\title{
Unsteady behavior of wall-detached flow inside a steam turbine control valve
}

Cite as: Phys. Fluids 31, 105101 (2019); https://doi.org/10.1063/1.5124359

Submitted: 13 August 2019 . Accepted: 18 September 2019 . Published Online: 02 October 2019

Peng Wang (王鹏) (D), Sihua Xu (徐嗣华), Li He (何力), and Yingzheng Liu (刘应征) (D)

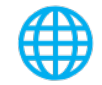

\section{ARTICLES YOU MAY BE INTERESTED IN}

Acoustics-driven vortex dynamics in channel branches with round intersections: Flow mode transition and three-dimensionality

Physics of Fluids 32, 025101 (2020); https://doi.org/10.1063/1.5141609

Notable effect of the subgrid-scale stress anisotropy on mean-velocity prediction through budget of the grid-scale Reynolds-shear stress

Physics of Fluids 31, 105103 (2019); https://doi.org/10.1063/1.5121528

Study on flow separation and transition of the airfoil in low Reynolds number

Physics of Fluids 31, 103601 (2019); https://doi.org/10.1063/1.5118736

Physics of Fluids Special Issue on the Lattice Boltzmann Method 


\title{
Unsteady behavior of wall-detached flow inside a steam turbine control valve
}

\author{
Cite as: Phys. Fluids 31, 105101 (2019); doi: 10.1063/1.5124359 \\ Submitted: 13 August 2019 • Accepted: 18 September 2019 • \\ Published Online: 2 October 2019
}

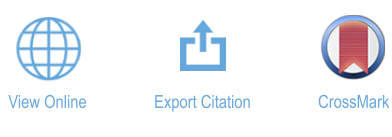

\author{
Peng Wang (王鹏), ${ }^{1,2,3}$ (D) Sihua Xu (徐嗣华), ${ }^{4}$ Li He (何力), ${ }^{3}$ and Yingzheng Liu (刘应征) ${ }^{1,2, a)}$ (D)

\begin{abstract}
AFFILIATIONS
${ }^{1}$ Key Laboratory for Power Machinery and Engineering of Ministry of Education, School of Mechanical Engineering, Shanghai Jiao Tong University, 800 Dongchuan Road, Shanghai 200240, China

${ }^{2}$ Gas Turbine Research Institute, Shanghai Jiao Tong University, 800 Dongchuan Road, Shanghai 200240, China

${ }^{3}$ Department of Engineering Science, University of Oxford, Parks Road, Oxford OX1 3PJ, United Kingdom

"Shanghai Turbine Plant, Shanghai Electric Power Generation Equipment Co., Ltd., 333 Jiangchuan Road, Shanghai 200240, China
\end{abstract}

a) Author to whom correspondence should be addressed: yzliu@sjtu.edu.cn

\begin{abstract}
Wall-detached flow inside an ultra-supercritical steam turbine control valve was comprehensively investigated with detached-eddy simulation, proper orthogonal decomposition (POD), and flow reconstruction. The dependency of the wall-detached flow on the control valve's opening ratio and pressure ratio was established first. Scattered wall-detached-flow, merged wall-detached-flow, and intersected wall-detached-flow were then identified by distinguishing the detachment scale of the wall-detached jet. Subsequently, flow analysis was conducted in terms of the statistical flow quantities, i.e., velocity fluctuation, turbulent kinetic energy, pressure loss, and pressure fluctuation. The statistical results demonstrated that the merged wall-detached-flow facilitated the most intensive velocity and pressure fluctuations inside the steam turbine control valve. The intersected wall-detached-flow encountered significant shock-wave reflections along the downstream pipe. By conducting POD analysis and flow reconstruction on the instantaneous flow snapshots, the dominant vortex structures and energetic pressure fluctuation modes were extracted to illustrate the wall-detached flow's unsteady behavior. The results showed that the instabilities of the scattered wall-detached-flow were primarily represented by the horizontal flapping motion of the wall-detached jet. However, for the merged wall-detached-flow, both the vertical out-phase oscillation and the horizontal flapping motion of the wall-detached jet intensified, yielding essential axial pressure fluctuation modes. As for the intersected wall-detached-flow, due to the complex wave reflections and propagations, essential regions with velocity discontinuities and diagonal crosslines with intensive pressure fluctuations formed inside the valve pipe. These findings are of great practical significance for the operation and optimization of steam turbine control valves in thermal power plants.
\end{abstract}

Published under license by AIP Publishing. https://doi.org/10.1063/1.5124359

\section{NOMENCLATURE}

\section{Main symbols}

$a_{\text {in }} \quad$ local sound speed at the valve inlet

$A_{n} \quad$ temporal coefficient of POD mode

$D_{\text {in }} \quad$ hydraulic diameter of the valve inlet

$D_{v} \quad$ diameter of the valve cavity

$l_{\max } \quad$ maximum lift distance of the valve spindle

$l_{v} \quad$ lift distance of the valve spindle

$M \quad$ Mach number

$n \quad$ order of POD mode

$\Delta p \quad$ pressure loss $p_{\text {in }}^{*} \quad$ total pressure at the valve inlet

$\bar{p}_{\text {out }} \quad$ averaged static pressure at the valve outlet

$\Delta p_{\text {out }} \quad$ pressure loss at the valve outlet

$P_{P O D} \quad$ pressure fluctuation of POD mode

$p_{r m s} \quad$ root mean square of pressure fluctuations

$R e_{\text {in }} \quad$ mainstream Reynolds number

St Strouhal number

$t_{i} \quad$ time interval of intersected wall-detached-flow

$t_{m} \quad$ time interval of merged wall-detached-flow

$t_{s} \quad$ time interval of scattered wall-detached-flow

$T_{\text {in }}^{*} \quad$ total temperature at the valve inlet

$U_{\text {in }} \quad$ mainstream velocity at the valve inlet 
$v_{P O D} \quad$ vertical velocity of POD mode

$v_{\text {rec }} \quad$ vertical velocity of reconstructed flow field

$v_{r m s} \quad$ root mean square of vertical velocity fluctuations

$y^{+} \quad$ nondimensional wall distance

\section{Greek symbols}

$\varepsilon \quad$ open ratio of the control valve

$\pi \quad$ pressure ratio of the control valve

$\rho_{\text {in }} \quad$ steam density at the valve inlet

$\mu_{\text {in }} \quad$ steam viscosity at the valve inlet

$\lambda_{n} \quad$ eigenvalue of POD mode

$\varphi_{n} \quad$ spatial feature of POD mode

\section{Abbreviations}

2D two-dimensional

3D three-dimensional

DES detached-eddy simulation

LES large-eddy simulation

POD proper orthogonal decomposition

Q2D quasi-two-dimensional

RANS Reynolds-averaged Navier-Stokes

RMS root mean square

SAS scale-adaptive simulation

SST shear stress transport

TKE turbulent kinetic energy

\section{INTRODUCTION}

As the major flow control component in modern thermal power plants, steam turbine control valves are commonly placed between the boiler and the steam turbine to manipulate the superheated steam flow rate for the plant's flexible operation. In practice, the control valves are inevitably restricted to work at limited opening ratios with considerable pressure drops. Especially for the part-load running, preheating, start-up, and shut-down processes of the steam turbine system, the high-pressure mainstream steam flow is strongly accelerated, forming supersonic jets near the valve throat. As the supersonic jets detach from the valve wall and impinge into the downstream flow passage, very complex flow patterns with intensified pressure fluctuations are developed, which usually results in unexpected aerodynamic noises, ${ }^{1,2}$ structural vibrations, ${ }^{3}$ and even fatigue failures. ${ }^{4}$ Accordingly, a comprehensive understanding of the unsteady behaviors of the wall-detached flow inside the control valve is highly desirable for aerodynamic design and system safety.

A literature survey shows that many efforts have been made to clarify the flow patterns inside steam turbine control valves. Hardin et $a .^{3}$ and Michaud et al. ${ }^{4}$ reported serious accidents of valve vibrations and pipe fatigue failures in power plants, which were confirmed to be highly related to unstable flow separation inside the control valves. To investigate an aerodynamic noise elevation of $10 \mathrm{~dB}$, Nakano et al. ${ }^{5,6}$ used schlieren visualization to identify the cause as the jet flow's detachment from the valve seat to the valve spindle, which was strongly coupled with complex shock-boundary layer interactions. Pluviose identified unstable wall-detached flow and shock-wave propagation inside the downstream pipe using a quasi-two-dimensional (Q2D) simplified valve model. Based on the frequency and amplitude of the measured pressure pulsations, Zhang et al. ${ }^{8,9}$ classified the valve's flow pattern into the annular wall-attached flow, the axisymmetric free supersonic jet flow, the uniform flow, and the asymmetric wall-detached flow. Among all patterns, the asymmetric wall-detached flow gave rise to the most unstable pressure fluctuations. Zeng et al. ${ }^{10}$ demonstrated that the transition from wall-attached flow to wall-detached flow gave rise to the phenomenon of sound mutation inside the control valve. Recently, Domnick et al. ${ }^{11}$ performed numerical simulations of valve flow using the shear stress transport (SST) turbulence model and established the dependencies of wall-attached flow and walldetached flow on the control valve's opening ratio and pressure ratio; their results showed that the transition from wall-attached flow to wall-detached flow was related to the Coandă effect, which tended to occur under intermediate opening ratios. Wang et al. ${ }^{12}$ also performed steady-state SST simulations and illustrated wall-attached flow and wall-detached flow inside a steam turbine control valve. From these studies, the qualitative patterns of the wall-attached flow and wall-detached flow are illustrated in Fig. 1. The wall-attached flow is mainly represented by the surrounding wall-attached jet and central reverse flow, whereas wall-detached flow is primarily represented by the central wall-detached jet, surrounding reverse flow, and reversed cavity vortex flow.

Furthermore, unsteady flow behaviors inside the control valves have been investigated through high-fidelity numerical simulations such as scale-adaptive simulation (SAS), detached-eddy simulation (DES), and large-eddy simulation (LES). For traditional valves with a spherical spindle, Morita et al. ${ }^{13}$ performed LES on a scaled-down model, and they found that the unstable wall-detached flow induced a cyclic asymmetric side load to the valve spindle; the SAS of Zanazzi et $a l^{14}$ showed that unstable wall-detached flow also resulted in severe shock-wave oscillations inside the downstream pipe. Subsequently, Yonezawa et al. ${ }^{15}$ conducted DESs with experimental validation for a cutoff control valve; their numerical results demonstrated that wall-detached flow introduced a rotating high-pressure region acting on the valve spindle, yielding a periodically fluctuating lateral force. Similarly, Zeng et al. ${ }^{16,17}$ reported that a high-pressure region, which acted on the valve spindle under intermediate opening ratios, yielded low-frequency intermittent vibrations of the valve. More recently, a bell-shaped valve spindle was specially developed to attenuate rotating-pressure-induced vibrations. In this regard, Domnick et al. ${ }^{18,19}$ conducted a scale-adaptive simulation with an embedded force (SAS-F) to determine the transient flow fields and showed that wall-attached flow induced weak turbulent flow fluctuations, whereas wall-detached flow gave rise to strong broad fluctuations. From DESs of the unsteady behaviors of wall-attached flow, in-phase and out-phase oscillations of the annular wall-attached jet were successfully identified by state-of-the-art proper orthogonal decomposition (POD). ${ }^{20,21}$ However, comprehensively understanding the wall-detached flow is still insufficient, especially the dominant vortex structures and energetic pressure fluctuations, which contribute mostly to the highly unstable three-dimensional (3D) flows inside steam turbine control valves. ${ }^{22,23}$

Continuing a previous study by Wang et al., ${ }^{22}$ the present study focuses primarily on the unsteady behavior of wall-detached flow inside a bell-shaped steam turbine control valve. To this end, the dependency of wall-detached flow inside an ultra-supercritical steam turbine $^{24}$ control valve on the valve's opening ratio and pressure 
(a)

\section{$\longrightarrow$ Annular wall-attached jet Central reverse flow}

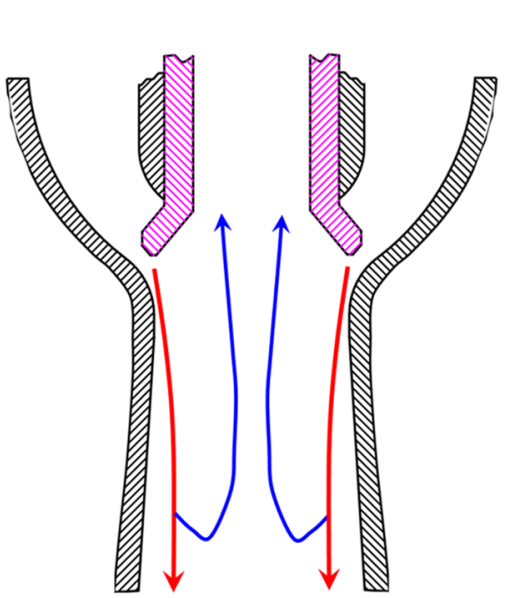

(b)
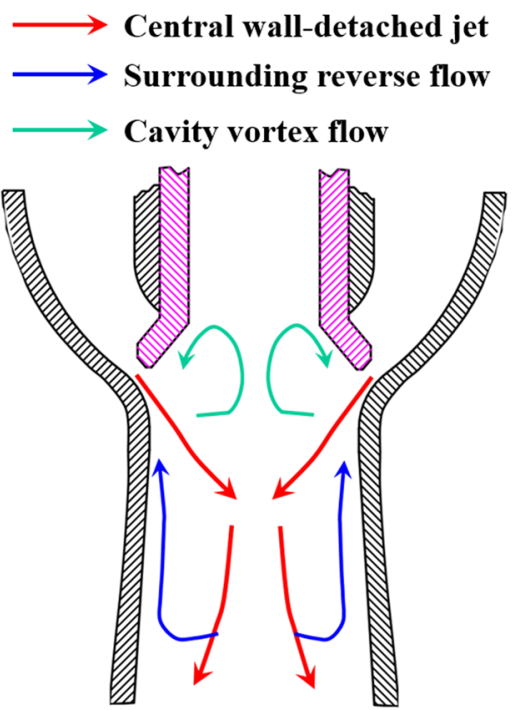

FIG. 1. Schematic of the (a) wall-attached flow and (b) walldetached flow inside a steam turbine control valve. ratio was established first. Subsequently, the unsteady flow dynamics of the wall-detached flow were numerically simulated with DES; the boundary conditions were identical to those in the power plant. The unsteady flow dynamics of the wall-attached flow were also simulated for comparison.

This paper is organized as follows. In Sec. II, the configuration of the control valve and the numerical setup of the DES are described. In Sec. III A, the wall-attached flow and wall-detached flow are classified under a wide range of valve-openings and pressure ratios. The statistical flow characteristics of the wall-detached flow are illustrated in Sec. III B. Finally, in Sec. III C, the dominant vortex structures and energetic pressure fluctuation modes of the wall-detached flow are extracted from the POD analysis of the velocity field and pressure field, respectively.

\section{NUMERICAL SETUP OF THE STEAM TURBINE CONTROL VALVE}

Schematics of the structural components of a domestic ultrasupercritical steam turbine control valve are illustrated in Fig. 2. In thermal power plants, the superheated steam flow arising from the upstream boiler directly enters the control valve and exhausts into the downstream steam turbine chamber. The structural configuration of the control valve comprises a moving spindle and other fixed components, i.e., a valve chamber, a valve casing, and a valve pipe. Because the valve spindle is bell-shaped, valve-induced pressure fluctuations can be prevented from acting directly on the inner wall surfaces, achieving significant attenuation of flow-induced vibrations. In practice, the displacement of the valve spindle is controlled by a high-precision hydraulic actuator that flexibly adjusts the opening ratio of the valve in response to varying power output. The fixed valve casing, along with some rubber seal rings, is arranged to guarantee the spindle's translational movements along its axis. In addition, a curved valve seat is installed opposite to the leading edge of the valve spindle, yielding a convergent-divergent flow passage, wherein the steam gas is initially compressed and then expanded. Immediately after the valve seat, a lengthy circular pipe is installed to attenuate the energetic pressure fluctuations before exhausting into the steam turbine chamber. However, these complex combinations result in a meandering flow passage inside the control valve, wherein the steam flow is undoubtedly modulated, exhibiting a large turbulence intensity.

In the present study, DES was used to determine the unsteady behavior of the wall-detached flow inside the control valve as the classical Reynolds-averaged Navier-Stokes (RANS) model totally failed to capture the fluctuated forces acting on a scaled valve model, ${ }^{18}$ whereas LES is too time-consuming for such high Reynolds-number flow inside such a complex valve configuration. The DES model was first proposed by Spalart et al. ${ }^{25}$ and has received widespread application in industrial aerodynamics, especially applications with high Reynolds numbers. This model can efficiently combine RANS with LES formulations; the RANS model is triggered around the wall surfaces, whereas the LES method is activated in the mainstream flow regions. Therefore, improved computational efficiency can be achieved compared with the LES method and higher numerical accuracy is gained compared with the RANS model. ${ }^{26}$ Note that in the region without flow unsteadiness, LES would not be triggered, while the flow field was modeled with RANS formulation. Using DES to simulate the complex valve flow has been validated by Yonezawa et al., ${ }^{15}$ where the frequencies of valve-induced pressure fluctuations were accurately captured and the rotating high-pressure region was confirmed to account for the flow-induced vibrations. Furthermore, according to Wang and Liu, ${ }^{20}$ who conducted DESs on unsteady flow behavior in a full-scale steam turbine control valve under the cold-start warming-up condition of the steam turbine system, the frequencies of the fluctuating forces acting on the valve spindle determined by the DES agreed quite well with the field data measured in power plants. Similarly with the study by Wang and Liu, ${ }^{20}$ the SST-based DES approach was also adopted in the present study. 
(a)

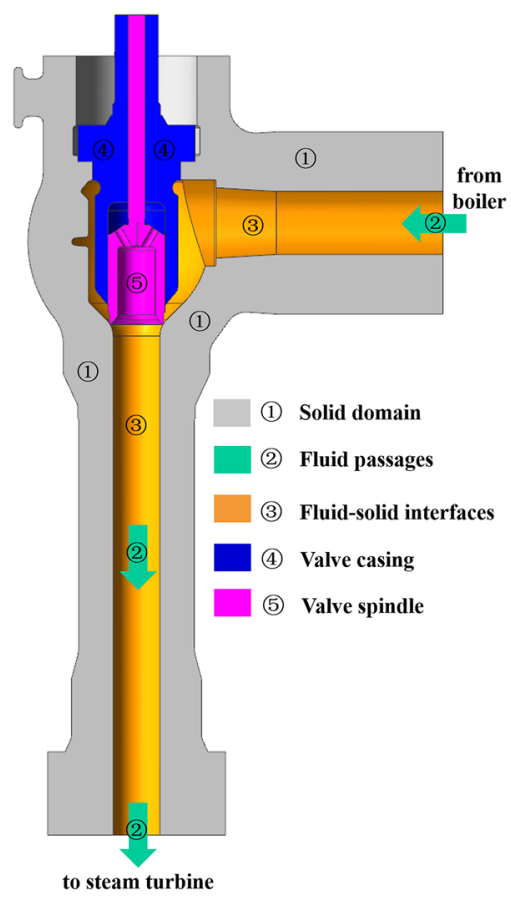

(b)

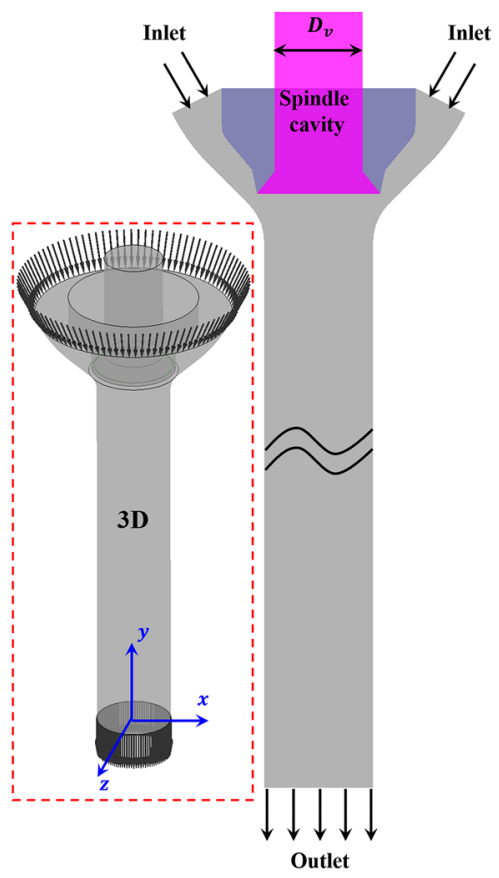

FIG. 2. Schematics of (a) the solid structural components and (b) the corresponding fluid domain of the steam turbine control valve.
In the current study, the fluid domain of the control valve [Fig. 2(b)] was extracted from the complex solid valve components and simplified into a $3 \mathrm{D}$ axisymmetric model for DESs. In the numerical simulations, the boundary conditions were identical to those of the power plant, and the operational data were extracted from the digital electric hydraulic (DEH) system of the steam turbine. The total pressure $\left(p_{i n}^{*}=26.25 \mathrm{MPa}\right)$ and total temperature $\left(T_{i n}^{*}=600{ }^{\circ} \mathrm{C}\right)$ of the mainstream steam flow at the valve inlet were

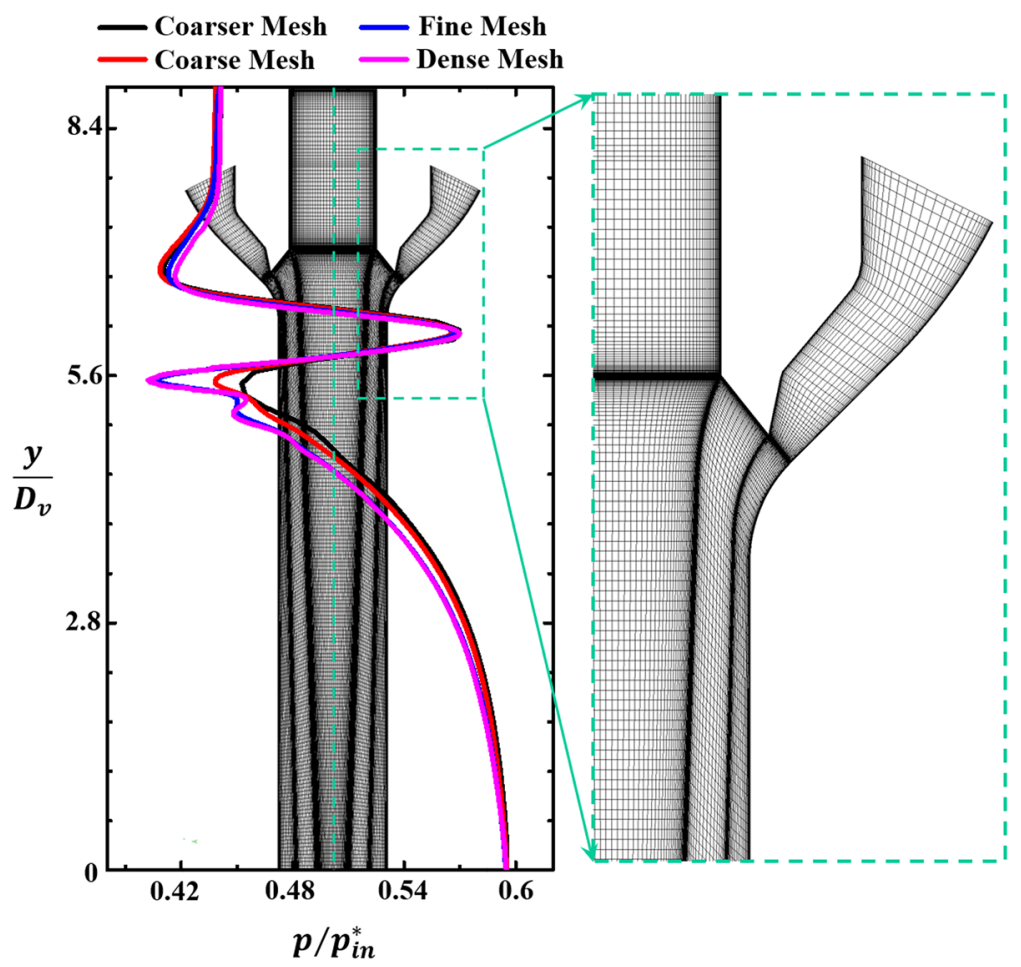

FIG. 3. Results of the mesh independence test and the multiblock well-organized mesh distribution inside the fluid domain of the steam turbine control valve. 
kept constant. The averaged static pressure at the valve outlet $\left(\bar{p}_{\text {out }}\right)$ was also kept constant. The pressure ratio of the control valve is defined as $\pi=\bar{p}_{\text {out }} / p_{\text {in }}^{*}$. In addition, the translational displacement of the valve spindle was kept fixed, resulting in a constant opening ratio $\left(\varepsilon=l_{v} / l_{\max }\right)$. Here, $l_{v}$ is the lift distance of the valve spindle and $l_{\max }$ is the maximum lift distance. The intermediate opening ratios $(0.2 \leq \varepsilon \leq 0.6)$, which have been demonstrated most probably to produce wall-detached flow, were selected for demonstration. ${ }^{11}$

Furthermore, a mesh independence test was conducted, and profiles of the time-averaged pressure along the centerline of the valve with $\varepsilon=0.5$ and $\pi=0.6$ were extracted from the DESs with different mesh sizes. As shown in Fig. 3, a fine mesh was eventually selected to discretize the valve's fluid domain, and almost $6 \times 10^{6}$ hexahedral mesh nodes with a multiblock well-organized distribution were generated. The spacing of the first cell along the valve's wall surfaces was set as $0.01 \mathrm{~mm}$. Most of the normalized wall distances $\left(y^{+}\right)$were below 50, while the maximum $y^{+}$reached 500 around the valve throat, where the steam flow was supersonic. A wall function was applied for near-wall treatment. The IAPWS-IF97 real gas model ${ }^{27}$ was used to determine the properties of the superheated steam fluid. The time step for the unsteady DESs was set at $0.00001 \mathrm{~s}$. The simulations were conducted using the commercial software package ANSYS CFX 16.0 with a fully coupled implicit solver. A second-order accurate high-resolution advection scheme and an implicit second-order accurate time differencing scheme were used to solve the NS equations. ${ }^{28}$ The well-converged flow fields, which were determined from steady-state simulations with the SST turbulence model, were used as the initial flow fields of corresponding DESs. An approximately constant transient level of fluctuating pressure was reached after $0.06 \mathrm{~s}$, and the physical time was about $0.25 \mathrm{~s}$. It took over 50 days to complete each DES with a parallel running on a workstation with $36 \mathrm{CPU}$ cores $(3.1 \mathrm{GHz})$.

\section{RESULTS AND DISCUSSION}

\section{A. Flow pattern classification}

To classify the flow patterns inside the steam turbine control valve, two-dimensional (2D) steady-state SST simulations were preliminarily performed with valve-opening ratios $(\varepsilon)$ of $0.2-0.6$ and pressure ratios $(\pi)$ of $0.8-0.25$, yielding the mainstream Reynolds number $\left(R e_{i n}=\frac{\rho_{i n} U_{i n} D_{i n}}{\mu_{i n}}\right)$ ranging from $R e_{i n}=1.38 \times 10^{7}$ to $R e_{i n}$ $=5.18 \times 10^{7}$. Here, $\rho_{i n}, \mu_{i n}$, and $U_{\text {in }}$ are the density, dynamic viscosity, and velocity of the mainstream steam gas at the valve inlet, respectively; $D_{i n}$ is the hydraulic diameter of the valve inlet. For each fixed opening ratio, a sequence of simulations was performed with gradually decreasing pressure ratios; simultaneously, the flow field from each preceding simulation was used as the initial condition for the current simulation. The dependencies of the wall-attached flow and wall-detached flow on the valve's opening ratio and pressure ratio are summarized in Fig. 4. For the wall-attached flow pattern, due to the Coandă effect, ${ }^{29-31}$ the high-speed annular jet that forms at the valve throat is continually attaching to the surrounding wall surfaces when impinging into the lengthy circular pipe of the valve. As a result, massive reverse flows are produced in the core region of the circular pipe. The unsteady flow behaviors of such wall-attached flow, i.e., the in-phase and out-phase oscillations of the annular wall-attached jet and the intermediate shear layer instabilities, were

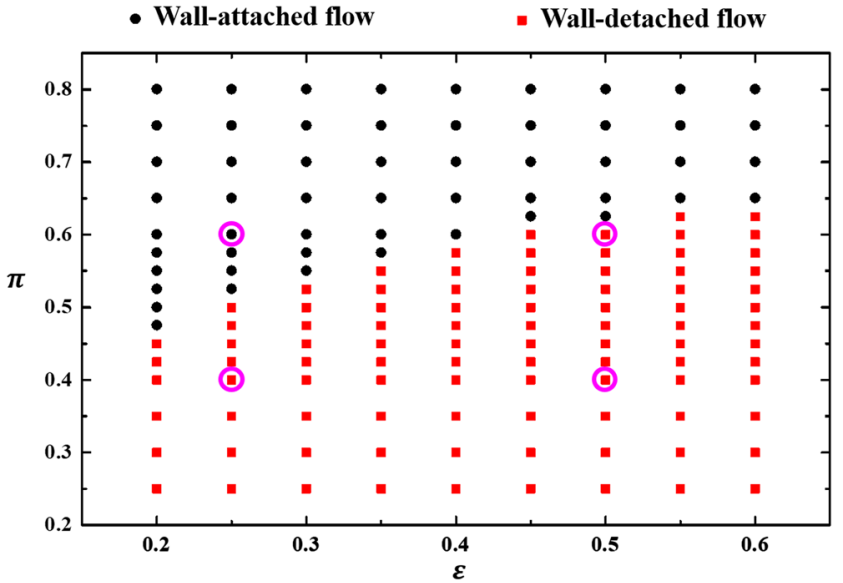

FIG. 4. Dependencies of the wall-attached flow and wall-detached flow on the opening ratio and pressure ratio of the steam turbine control valve.

reported in detail by Wang et al. ${ }^{22}$ However, the wall-detached flow pattern generally appears under more extreme flow conditions with larger opening ratios or lower pressure ratios and thus may generate more severe aerodynamic problems in the valve system. In such a flow pattern, the supersonic jet would detach from the surrounding wall surfaces and directly impinge into the core region of the downstream pipe. Due to the turbulent entrainment effect, the surrounding flow would be reversed along the wall surfaces, resulting in shear layer instabilities between the reverse flow and the walldetached jet. $^{32,33}$ A pair of energetic vortex structures would also be produced around the entrance of the deep spindle cavity, which may introduce flow instabilities inside the cavity. Beyond that, the opening ratio and pressure ratio would also affect the detachment scale of the high-speed jet, yielding different flow behaviors. Consequently, it is necessary to quantify the underlying flow dynamics of the wall-detached flow inside the steam turbine control valve.

To demonstrate the flow dynamics of the wall-detached flow, 3D transient-state DESs were conducted under the flow conditions marked in Fig. 4. Among these flow conditions, $\varepsilon=0.25$ and $\pi=0.6$ were related to the wall-attached flow, which served as a baseline. The other three flow conditions corresponded to different walldetached flow patterns, which were identified by distinguishing the detachment scale of the central wall-detached jet: $\varepsilon=0.5$ and $\pi=0.6$ were related to the scattered wall-detached-flow; $\varepsilon=0.25$ and $\pi=0.4$ were related to the merged wall-detached-flow; and $\varepsilon=0.5$ and $\pi=0.4$ were related to the intersected wall-detached-flow. The corresponding time-averaged flow fields, presented by the Mach number and velocity vector distributions, are illustrated in Fig. 5. For scattered wall-detached-flow, the annular jets detached slightly from the surrounding wall surfaces, independently expanded toward the valve outlet without merging together inside the core of the circular pipe. However, for merged wall-detached-flow, the annular jets detached more severely from the surrounding wall surfaces and directly merged together inside the pipe core region. The merged jets then synchronously expanded to the valve outlet with a gradually attenuated Mach number distribution. In intersected wall-detachedflow, the larger opening ratio and lower pressure ratio generated 
(a)

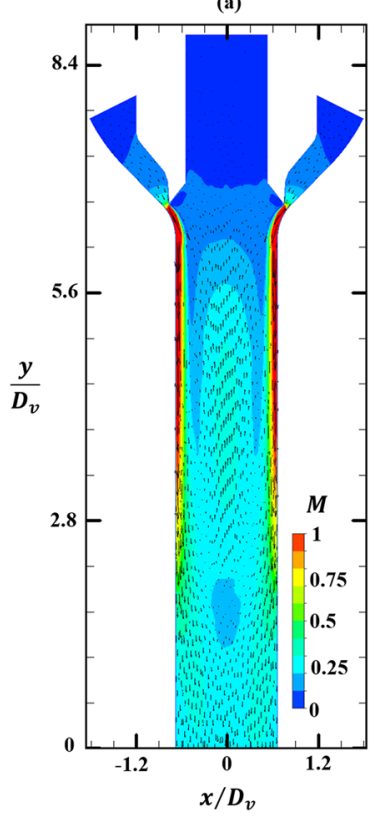

(b)

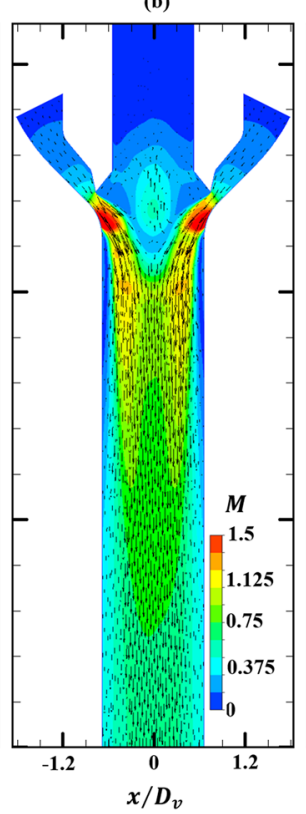

(c)

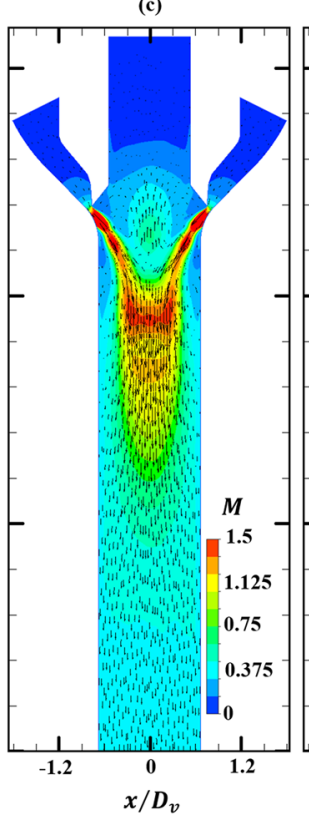

(d)

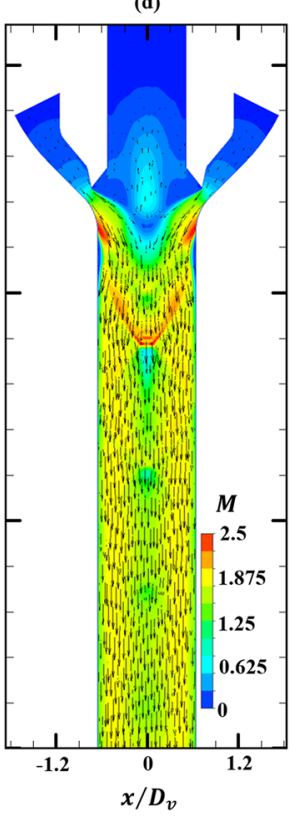

FIG. 5. Contour plots of the flow patterns (Mach number and velocity vector distributions) inside the steam turbine control valve: (a) Wall-attached flow $(\varepsilon=0.25$, $\pi=0.6)$; (b) scattered wall-detachedflow $(\varepsilon=0.5, \pi=0.6)$; (c) merged walldetached-flow ( $\varepsilon=0.25, \pi=0.4$ ); and (d) intersected wall-detached-flow $(\varepsilon=0.5$, $\pi=0.4$ ). a very high Mach number comparable to that of a supersonic jet around the valve throat. Thus, complex expansion waves, compression waves, and even shock waves appeared inside the control valve, as identified by the discontinuities of the Mach number distribution. The wave reflections on the wall surfaces of the circular pipe interacted with the jet expansions, ${ }^{34}$ yielding intersected flow fluctuations inside the pipe. Except for the different detachment scales of the central wall-detached jet, there is a common feature among these three wall-detached flow patterns: an elliptical zone with essential reverse flows located beneath the entrance of the deep spindle cavity. These reverse vortex flows could act on the valve spindle, inducing fluctuated aerodynamic forces and unexpected noise.

Subsequently, the $3 \mathrm{D}$ vortex structures inside the steam turbine control valve were discussed, and the merged wall-detachedflow was selected for demonstration in Fig. 6. Here, the instantaneous vortices were generated by the $Q$-criterion with $Q=0.001$ and (a)

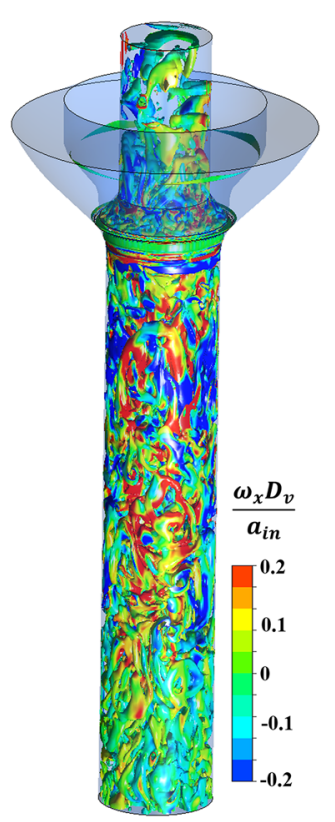

(b)

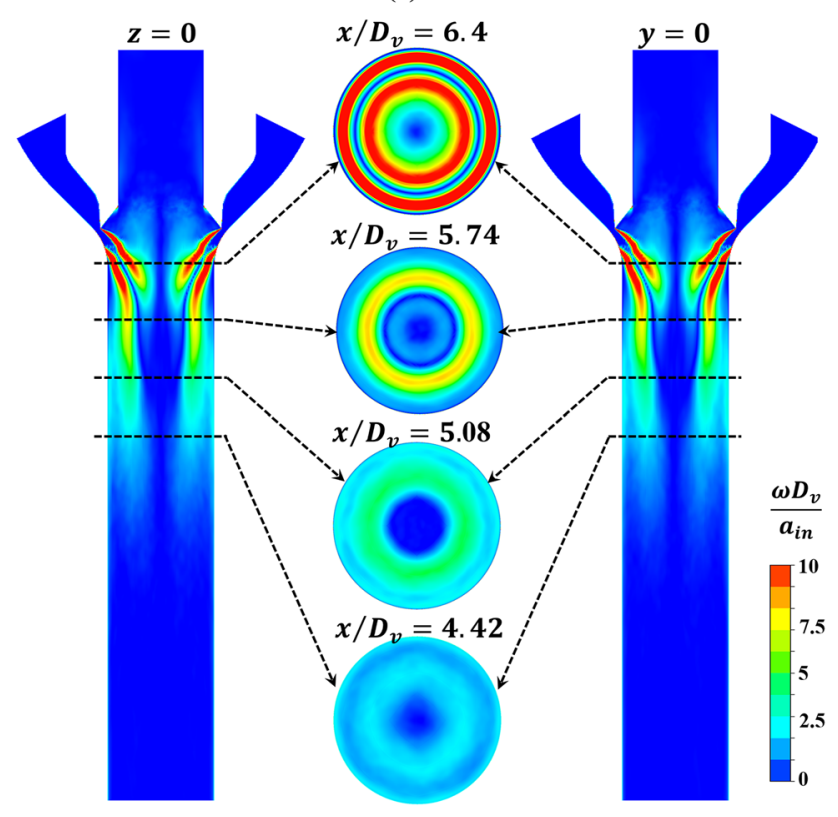

FIG. 6. Contour plots of the 3D vortex structures of the merged wall-detachedflow $(\varepsilon=0.25, \pi=0.4)$ inside the steam turbine control valve: (a) instantaneous vortices with $Q=0.001$ and (b) timeaveraged vorticity. 


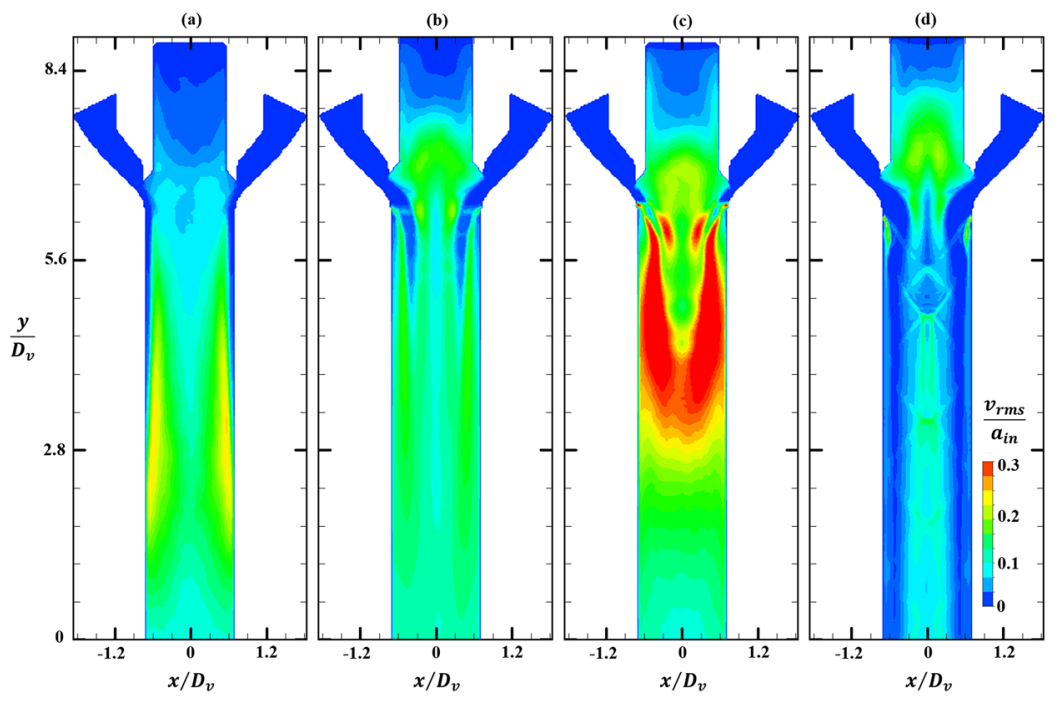

FIG. 7. Distributions of vertical velocity fluctuations inside the steam turbine control valve: (a) Wall-attached flow $(\varepsilon=0.25, \pi=0.6)$; (b) scattered wall-detached-flow $(\varepsilon=0.5$, $\pi=0.6)$; (c) merged wall-detached-flow $(\varepsilon=0.25, \pi=0.4)$; and (d) intersected wall-detached-flow $(\varepsilon=0.5, \pi=0.4)$.

characterized by the streamwise vorticity $\left(\omega_{x}\right)$, and the contour plots of time-averaged vorticity in different planes are also illustrated. Obviously, quite chaotic vortex structures with different scales and vorticities can be identified inside the steam turbine control valve. However, the time-averaged vorticity contours in Fig. 6(b) intuitively exhibit the axial symmetric feature of the 3D flow field. Six planes inside the steam turbine control valve were selected to illustrate the $3 \mathrm{D}$ flow field. In the planes of $z=0$ and $y=0$, a symmetric distribution of time-averaged vorticity can be identified. Two pairs of elongated regions with intensive vorticities are symmetrically distributed from the valve throat to the valve outlet. For the regions starting from the valve spindle, the intensive vorticities were attributed to the interactions between the wall-detached jet and the cavity vortex flow. However, for the regions starting from the valve seat, the intensive vorticities were attributed to the interactions between the wall-detached jet and the surrounding reverse flow. Furthermore, all the contour plots of time-averaged vorticity at planes of $x / D_{v}=6.4$, $5.74,5.08$, and 4.42 presented a highly circumferential-symmetric feature even though the vorticities were significantly decreased. Note that for the $3 \mathrm{D}$ vortex structures of the scattered wall-detached-flow and intersected wall-detached-flow, an axial symmetric feature can also be identified. Therefore, the flow information in the plane of $z=0$ was selected to investigate the statistical flow characteristics and dominant flow modes in Secs. III B and III C.
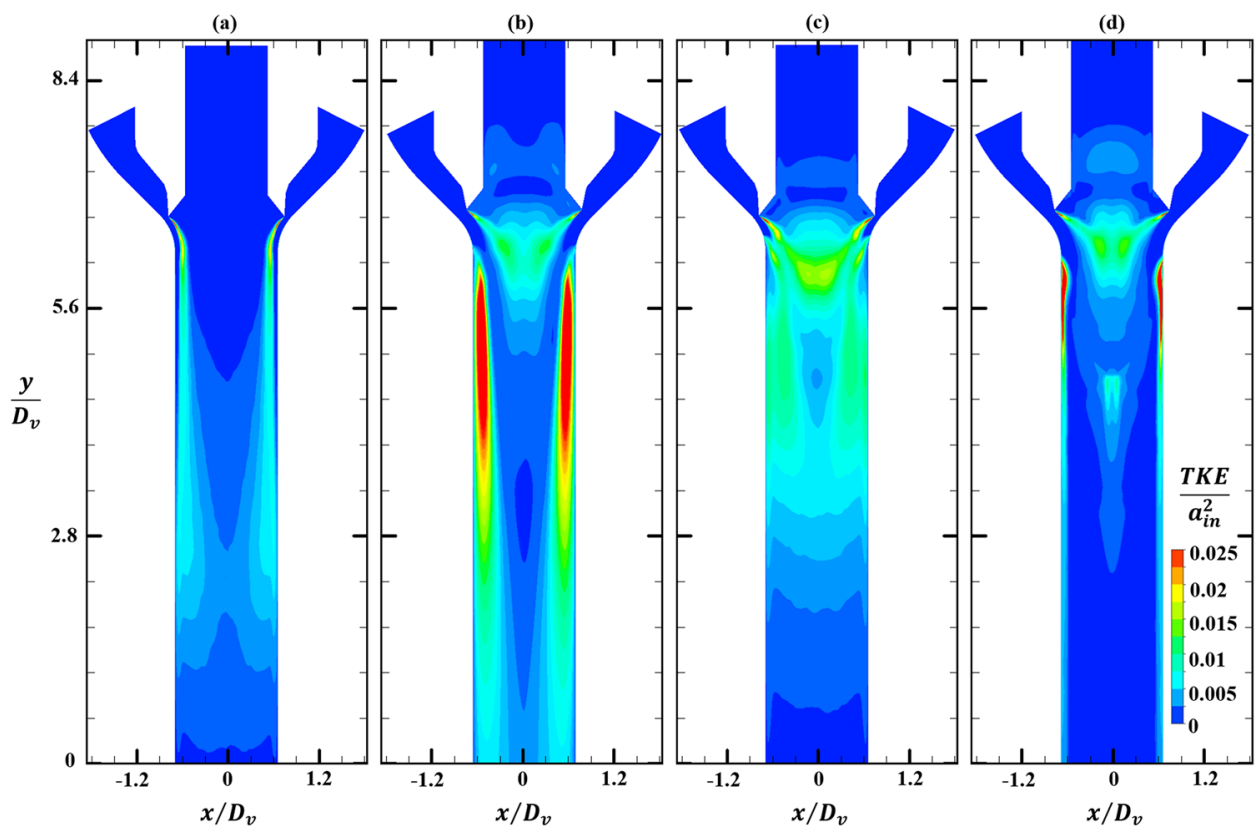

FIG. 8. Distributions of turbulent kinetic energy inside the steam turbine control valve: (a) Wall-attached flow $(\varepsilon=0.25$, $\pi=0.6$ ); (b) scattered wall-detachedflow $(\varepsilon=0.5, \pi=0.6)$; (c) merged walldetached-flow $(\varepsilon=0.25, \pi=0.4)$; and (d) intersected wall-detached-flow $(\varepsilon=0.5$, $\pi=0.4$ ). 

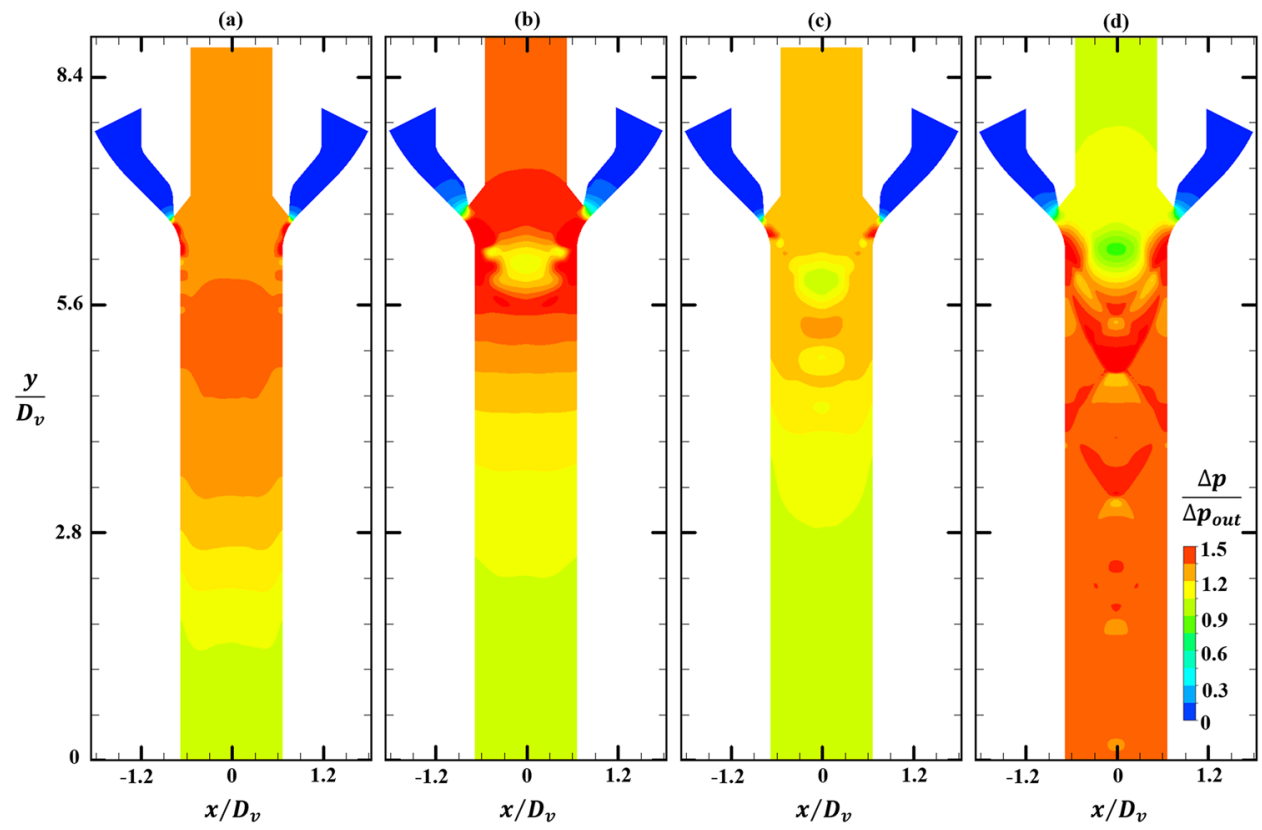

FIG. 9. Distributions of pressure loss inside the steam turbine control valve: (a) Wall-attached flow $(\varepsilon=0.25, \pi=0.6)$; (b) scattered wall-detached-flow $(\varepsilon=0.5$, $\pi=0.6$ ); (c) merged wall-detached-flow $(\varepsilon=0.25, \pi=0.4)$; and (d) intersected wall-detached-flow $(\varepsilon=0.5, \pi=0.4)$.

\section{B. Statistical flow characteristics}

To evaluate the statistical flow characteristics of the walldetached flows inside the steam turbine control valve, four statistical flow quantities, i.e., root mean square (RMS) of vertical velocity fluctuation, turbulent kinetic energy (TKE), pressure loss, and RMS of pressure fluctuation, were extracted from the instantaneous DES flow snapshots and are illustrated in this section.
RMS distributions of the vertical velocity fluctuations $\left(\frac{v_{r m s}}{a_{i n}}\right)$ inside the steam turbine control valve for scattered wall-detachedflow, merged wall-detached-flow, and intersected wall-detachedflow are illustrated in Fig. 7. Here, the RMS of vertical velocity fluctuation was normalized by the local speed of sound at the valve inlet $\left(a_{i n}\right)$. Obviously, the merged wall-detached-flow exhibited the most intensive velocity fluctuations. In particular, a pair of elongated regions with the most intensive velocity fluctuations was
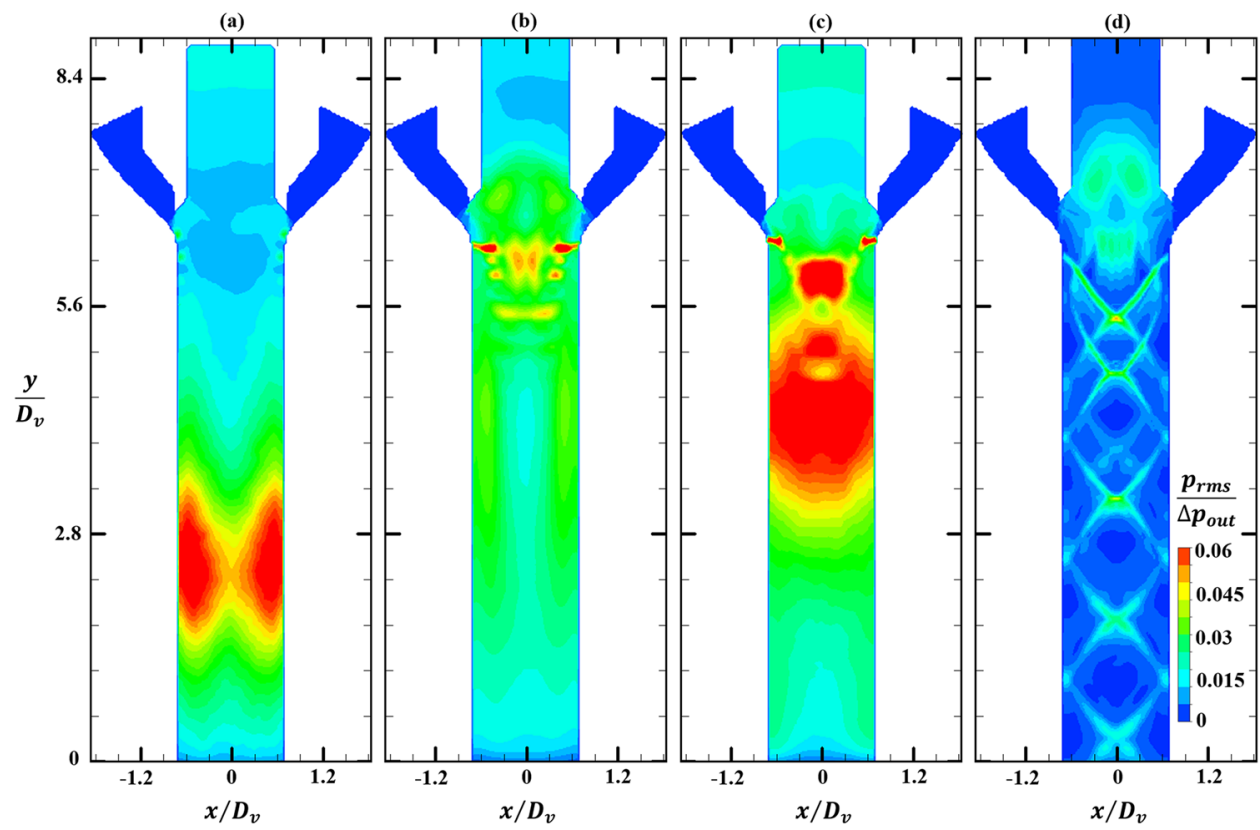

FIG. 10. Distributions of the pressure fluctuations inside the steam turbine control valve: (a) Wall-attached flow $(\varepsilon=0.25, \pi=0.6)$; (b) scattered walldetached-flow $(\varepsilon=0.5, \pi=0.6)$; (c) merged wall-detached-flow $(\varepsilon=0.25$, $\pi=0.4)$; and (d) intersected walldetached-flow $(\varepsilon=0.5, \pi=0.4)$. 
identified in the middle of the circular pipe $\left(2.8 \leq y / D_{v} \leq 6.2\right)$. These intensive velocity fluctuations are closely related to the interactions between the central wall-detached jet and the surrounding reverse flow. In addition, the velocity fluctuations corresponding to scattered wall-detached-flow were relatively attenuated and distributed uniformly inside the circular pipe. However, for intersected walldetached-flow, even the global magnitudes of the velocity fluctuations were relatively attenuated; a series of diagonal crosslines were identified inside the circular pipe, which are related to the complex wave reflections along the pipe. ${ }^{35}$ Furthermore, there is a common feature among these three wall-detached flow patterns: the intensive velocity fluctuations inside the spindle cavity, which are related to the unstable cavity vortex flow. Comparatively, the velocity fluctuations inside the spindle cavity for the wall-attached flow were quite attenuated; the intensive velocity fluctuations were attached to the middle wall surfaces of the circular pipe $\left(1.6 \leq y / D_{v} \leq 4.2\right)$. These intensive velocity fluctuations are related to the oscillations of the annular wall-attached jet and were gradually attenuated toward the valve outlet.

The flow quantity of TKE is a high-order measure to evaluate the fluctuating velocity field. Comparisons of the TKE distributions of these four flow patterns are illustrated in Fig. 8. Note that the TKE magnitude was normalized by the square of the local speed of sound at the valve inlet $\left(a_{i n}\right)$. For wall-attached flow, two elongated regions with intensive TKE magnitudes were identified along the wall surfaces of the circular pipe, which are attributed to the oscillations of the annular wall-attached jet. For the remaining regions, the TKE magnitudes were quite small, indicating stable flow fields. For the three wall-detached flow patterns, the dominant TKE distributions contained two portions: (1) elongated wall-attached regions, which are related to interactions between the wall-detached jet and the surrounding reverse flows, and (2) an inverted trapezoidal region around the entrance of the deep spindle cavity, which is related to the unstable cavity vortex flow. Different TKE magnitudes were identified among these three wall-detached flow patterns. For scattered wall-detached-flow, the TKE magnitudes at the two elongated wall-attached regions were extremely high, indicating strong shear layer instabilities between the central wall-detached jet with the surrounding reverse flow. However, the core region in the circular pipe exhibited quite small TKE magnitudes, indicating that the expansion of scattered wall-detached-flow was relatively stable. For merged wall-detached-flow, the spatial feature of the intensive TKE distribution was highly similar to that of the vertical velocity fluctuations [Fig. 7(c)], where the intensive TKE magnitudes were primarily located at the top and in the middle of the circular pipe $\left(3.1 \leq y / D_{v}\right.$ $\leq 6.5$ ). For the intersected wall-detached-flow, the TKE magnitudes were obviously attenuated, especially at the wall-attached regions.

The pressure loss inside the steam turbine control valve is a major concern in power plants, and it can be used to evaluate the economic efficiency of the operating conditions. Distributions of the time-averaged pressure loss corresponding to the four flow patterns are illustrated in Fig. 9. Here, the pressure loss $\left(\frac{\Delta p}{\Delta p_{\text {out }}}=\frac{p_{\text {in }}^{*}-p}{p_{\text {in }}^{*}-\bar{p}_{\text {out }}}\right)$ was normalized by the pressure loss at the valve outlet $\left(\Delta p_{\text {out }}=p_{\text {in }}^{*}-\bar{p}_{\text {out }}\right)$. A global view of Figs. 9(a)-9(c) demonstrates a similar spatial feature of the pressure loss distribution: the region with larger pressure loss was generally located at the top of the circular pipe, and then, the pressure loss became gradually attenuated along the circular pipe.
The sudden huge pressure drops at the top of the circular pipe are attributed to the energy consumption associated with the impingement of the wall-detached jet. Along with attenuated expansion of the central wall-detached jet, considerable pressure recovery was achieved at the valve outlet, which coincides with the Carnot diffuser flow. ${ }^{36}$ However, for intersected wall-detached-flow, the pressure loss inside the circular pipe was always high. These essential pressure losses were transferred to complex wave reflections and propagated inside both the control valve and the downstream steam turbine chambers.

Finally, the flow quantity of the pressure fluctuations, which can serve as excitation sources for the aerodynamic noises and aerodynamic forces acting on the valve spindle, was used to quantify the statistical flow characteristics of the wall-detached flow patterns. The RMS distributions of the pressure fluctuations $\left(\frac{p_{r m s}}{\Delta p_{o u t}}\right)$ are illustrated in Fig. 10. Here, the RMS of the pressure fluctuations was normalized by the pressure loss at the valve outlet. The merged wall-detachedflow exhibited the most intensive pressure fluctuations inside the circular pipe $\left(3.1 \leq y / D_{v} \leq 6.2\right)$. These essential pressure fluctuations are closely related to the unstable expansion of the merged

(a)

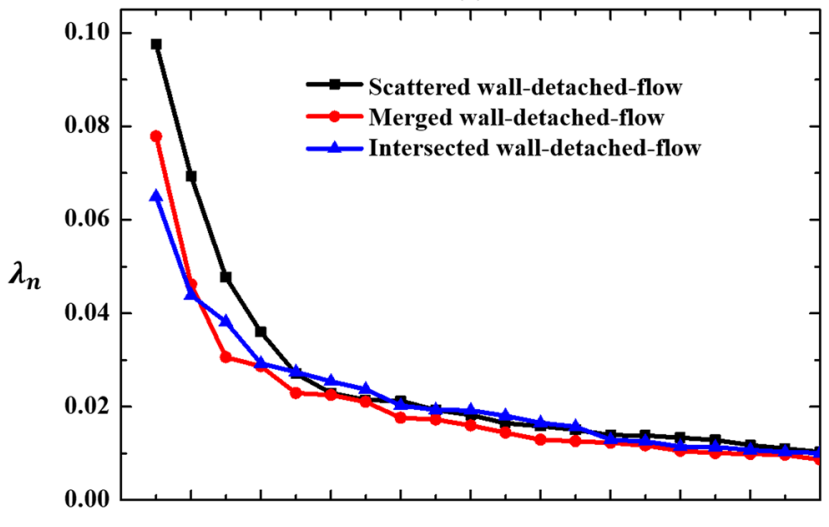

(b)

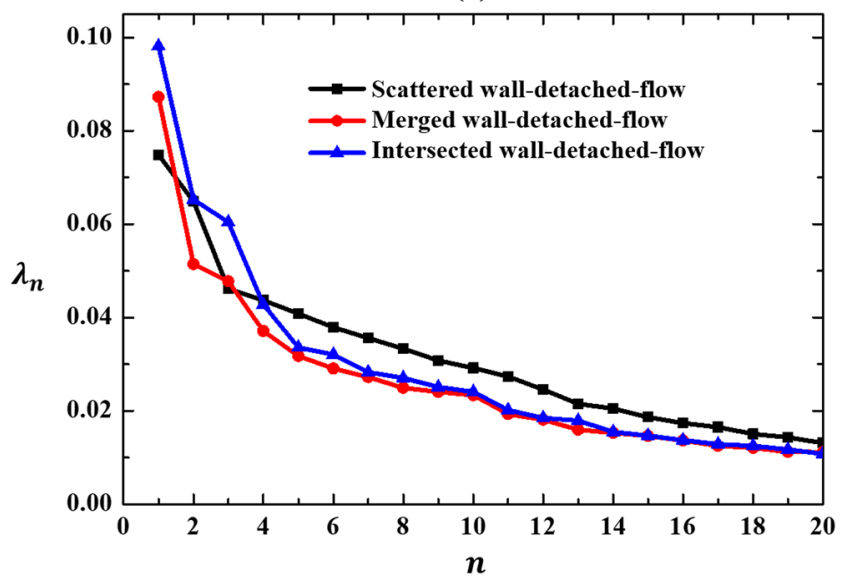

FIG. 11. Plots of normalized eigenvalues of the (a) velocity POD modes and (b) pressure POD modes. 
jets. Fortunately, the pressure fluctuations were gradually attenuated along the pipe centerline to the valve outlet. For the intersected wall-detached-flow, even the global pressure fluctuation intensity was attenuated compared with the merged wall-detached-flow; a series of diagonal crosslines with intensive pressure fluctuations were identified inside the circular pipe. These pressure fluctuations undoubtedly contribute to maintaining the unstable wave propagations inside the control valve $\mathrm{e}^{37}$ and may even be transferred to the downstream steam turbine systems, yielding dangerous structural problems. For the scattered wall-detached-flow, a uniform spatial feature of the pressure fluctuation was identified inside the circular pipe, which was similar to the velocity fluctuation distribution. Compared with the wall-attached flow, the pressure fluctuations at the bottom of the pipe were significantly attenuated for the scattered wall-detached-flow. However, the pressure fluctuations around the entrance of the spindle cavity, especially the small discrete regions, were significantly intensified. Once these pressure fluctuations acted on the valve spindle, severe flow-induced vibrations would result, just like the phenomenon that occurs in traditional control valves with spherical or cutoff spindles.

\section{Dominant flow modes}

A POD method was used to extract dominant flow modes of the wall-detached flows inside the steam turbine control valve. Generally, to conduct the POD analysis, a time-series of flow snapshots is ordered in a space-time cross correlation matrix. Then, eigenvalue decomposition is applied on this matrix, resulting in three pieces of information: the eigenvalue $\left(\lambda_{n}\right)$, which represents the relative contribution of each POD mode to the total fluctuation intensity of the flow field, the spatial feature of each POD mode $\left(\varphi_{n}\right)$, and the temporal coefficient $\left(A_{n}\right)$ of each POD mode. Here, $n$ is the order of POD mode. The dominant velocity/pressure fluctuation modes can be identified by ranking their eigenvalues, and the corresponding spatial distributions and temporal evolutions can be acquired from the spatial mode features and temporal mode coefficients. Details on the mathematical fundamentals of POD can be found in the work of Lumley $^{38}$ and Sirovich ${ }^{39}$ The flow reconstruction method, which combines the dominant velocity POD modes and the time-averaged flow field, is used as a complementary strategy incorporated in the POD method. With the flow reconstruction method, ${ }^{40}$ the
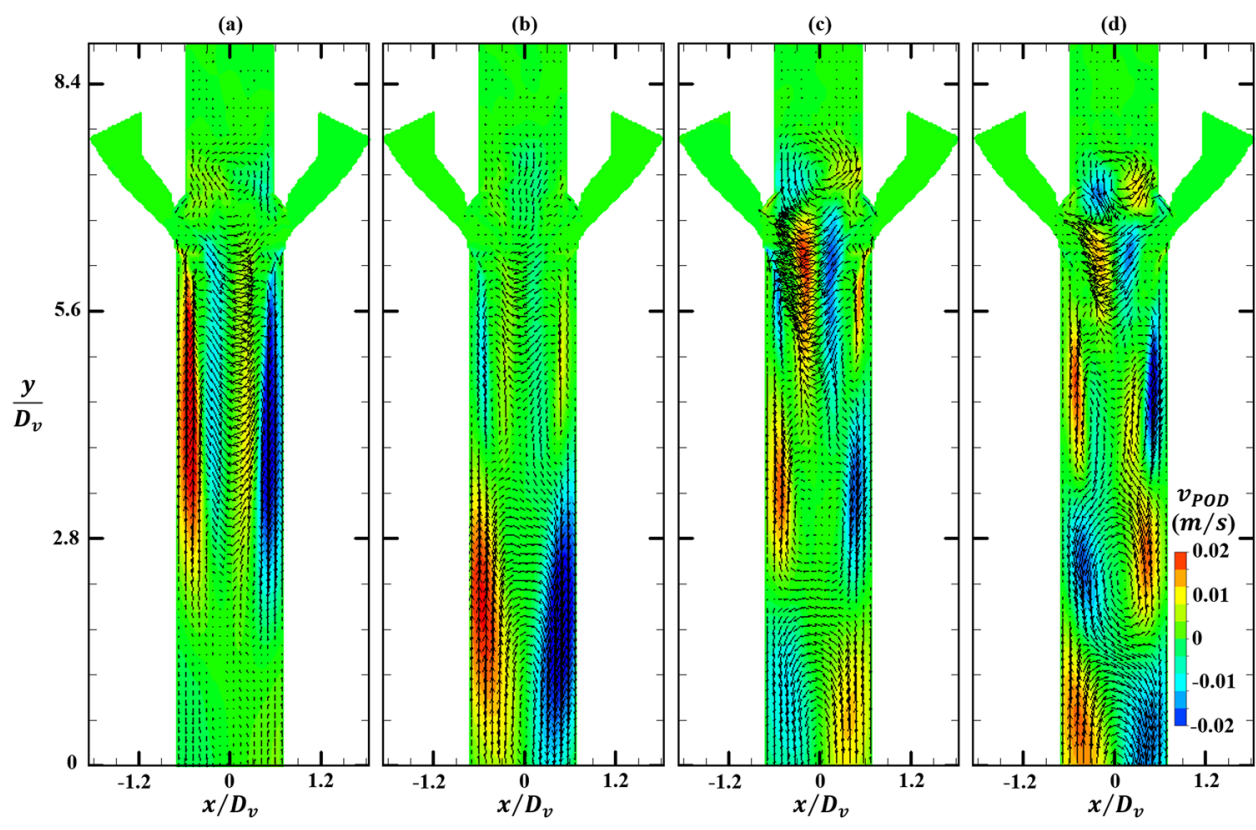

FIG. 12. Spatial patterns and the corresponding frequency spectra of the mode coefficients for the velocity POD modes of the scattered wall-detachedflow inside the steam turbine control valve $(\varepsilon=0.5, \pi=0.6)$ : (a) velocity POD mode 1 ; (b) velocity POD mode 2 ; (c) velocity POD mode 3 ; and (d) velocity POD mode 4.
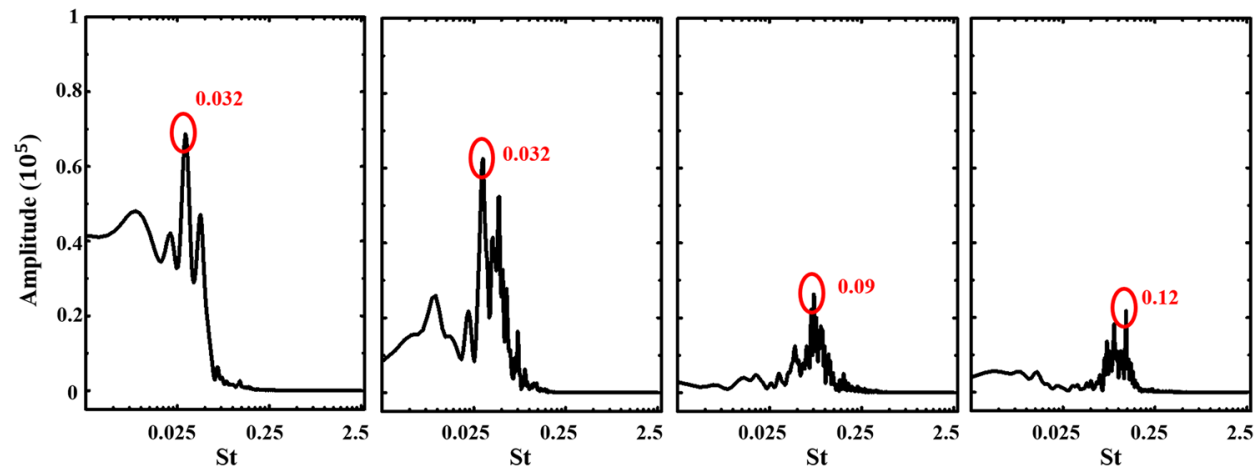
influence of dominant POD modes on the unsteady behaviors of the flow dynamics can be further investigated. The procedures for performing POD and flow reconstruction to analyze complex valve flows can be found in the work of Wang et al. ${ }^{21,22}$

POD analysis on the fluctuating velocity field and pressure field was conducted, and dominant velocity fluctuation modes and pressure fluctuation modes were successfully extracted. Figure 11 plots the normalized eigenvalues of the velocity POD and pressure POD modes. The magnitude of the eigenvalue $\left(\lambda_{n}\right)$ means the relative contribution of the eigenmode to the overall fluctuation intensity of the velocity field or pressure field. A similar trend was identified to all the eigenvalue plots: the eigenvalue decreased rapidly at the first several eigenmodes, indicating dominant contributions from these modes to the fluctuating flow fields. The eigenvalues of higher-order modes were much smaller, indicating attenuated contributions to the total flow fluctuation. In the present study, we focused primarily on the first four POD modes to illustrate the dominant flow structures of the wall-detached flows inside the steam turbine control valve. Simultaneously, the influence of the dominant vortex structures on the unsteady oscillations of the wall-detached flows was also investigated by reconstructing the velocity fields with dominant velocity POD modes.

\section{Scattered wall-detached-flow}

The spatial patterns and frequency spectra of the mode coefficients for the velocity POD modes of the scattered wall-detachedflow are displayed in Fig. 12. Here, the Strouhal number is defined as $S t=\frac{f D_{v}}{a_{\text {in }}}$, where $D_{v}$ is the diameter of the spindle cavity. A qualitative view of modes 1 and 2 shows a similar distribution, with massive positive and negative velocity fluctuations attached to the wall surfaces of the circular pipe, along with a large-scale clockwise vortex structure. This vortex structure and velocity fluctuations would undoubtedly act on the wall-detached jet, inducing the unsteady behaviors of the jet. However, evident differences in the locations of the vortex structure and velocity fluctuations were identified between these two modes. For mode 1, they were located at the top and in the middle of the valve pipe $\left(2.4 \leq y / D_{v} \leq 5.9\right)$, whereas for mode 2 , they were located at the bottom of the pipe $\left(0.2 \leq y / D_{v} \leq 2.9\right)$, where they would interact with the flow separations and reattachments at the trailing edges of the wall-detached jet. Modes 3 and 4 were recognized as the high-order harmonics of modes 1 and 2 as more regions with alternating positive and negative velocity fluctuations appeared along the valve pipe, along with the corresponding vortex structures. However, the velocity fluctuations and vortex structures around the entrance of the spindle cavity were more intensive than the first two modes. These velocity fluctuations would interact with the reversed cavity vortex flow, yielding flow instabilities inside the deep spindle cavity. A detailed inspection of the frequency spectra of the mode coefficients demonstrates the same peak frequency at $S t=0.032$ for the first two modes, whereas higher peak frequencies at $S t=0.09$ and $S t=0.12$ were identified for modes 3 and 4, respectively. Therefore, it is necessary to detect the influences of such correlated velocity modes on the unsteady behaviors of the scattered wall-detached-flow.

The first two velocity POD modes with similar spatial distributions and peak frequencies were used to reconstruct the flow field of scattered wall-detached-flow. Note that the corresponding timeaveraged flow field was also superimposed. Four successive phaseaveraged flow fields with intervals of $t_{s} / 4$ and average bins of $\pm 5^{\circ}$ were determined and are illustrated in Fig. 13. Here, $t_{s}$ corresponds to the peak frequency at $S t=0.032$, and the reconstructed vertical velocity $\left(v_{r e c}\right)$ and surface streamlines were used for demonstration.
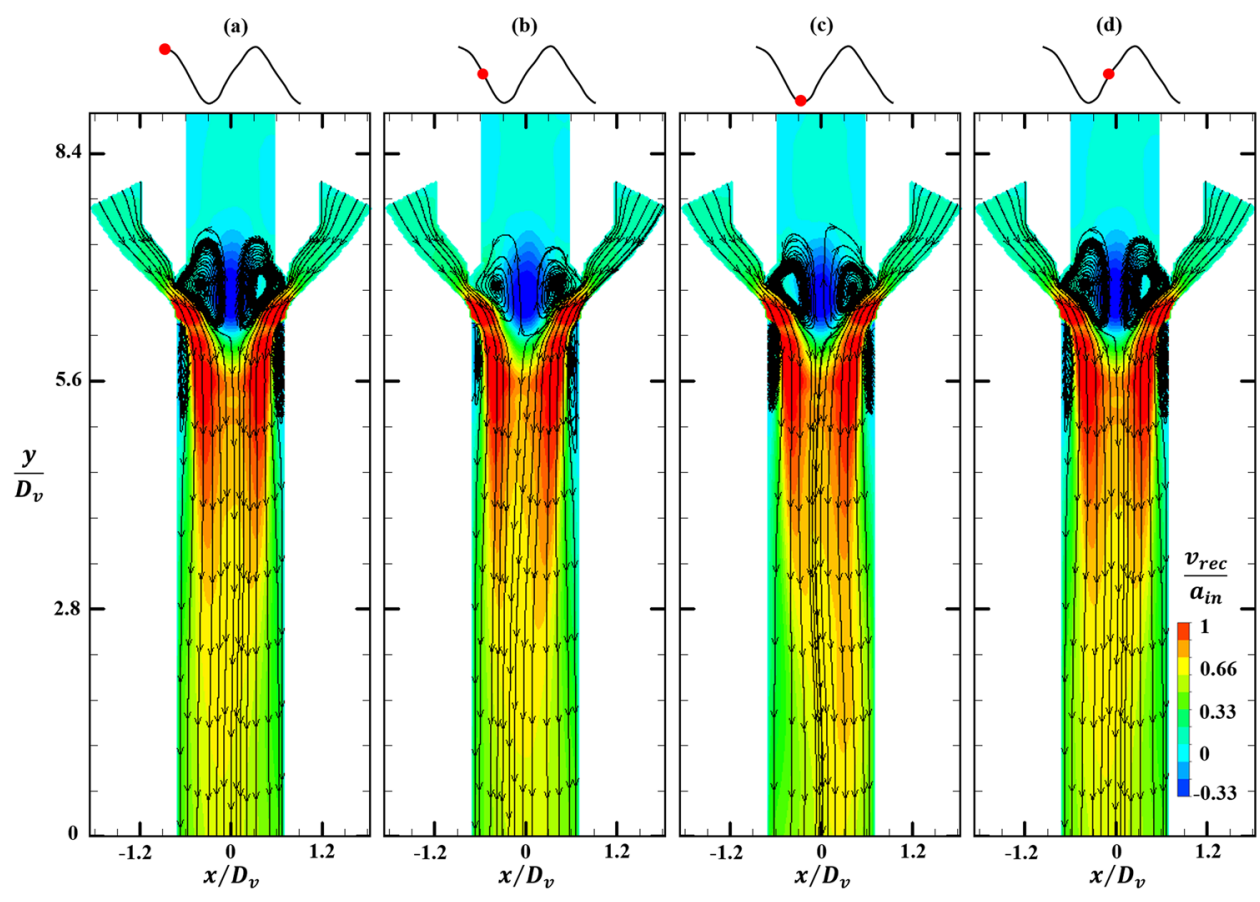

FIG. 13. Temporal evolutions of the reconstructed flow field with the first two velocity POD modes of the scattered wall-detached-flow $(\varepsilon=0.5, \pi=0.6)$ : (a) $t=0$; (b) $t=t_{s} / 4$; (c) $t=t_{s} / 2$; and (d) $t=3 t_{s} / 4$ 
Note that the surface streamlines were calculated with the reconstructed streamwise velocity $\left(u_{r e c}\right)$ and the reconstructed vertical velocity $\left(v_{r e c}\right)$ in this plane $(z=0)$. A global view of Fig. 13 demonstrates patterns with the time-averaged flow field similar to those shown in Fig. 5(b), i.e., the central wall-detached jet, the surrounding reverse flow, and the reversed cavity vortex flow. However, a comparative view of these four phase-dependent flow fields discloses the horizontal flapping motions of the wall-detached jet. At $t=0$, the left jet expanded more intensively than the right jet, and the trailing edges of the wall-detached jet flapped toward the left pipe edge. Subsequently, at $t=\frac{t_{s}}{4}$, the expansion of the right jet intensified, resulting in flapping of the trailing edges of the wall-detached jet to the centerline of the pipe. At $t=\frac{t_{s}}{2}$, the attenuation of the left-jet expansion along with the gradually intensified right-jet expansion aggravated the asymmetric distribution of the flow pattern such that the trailing edges of the wall-detached jet flapped toward the right pipe edge. Finally, at $t=\frac{3 t_{s}}{4}$, the right jet expansion attenuated significantly, whereas the left jet expansion intensified such that the trailing edges of the wall-detached jet flapped again to the centerline of the pipe. In addition, the surrounding reverse flow oscillated along with the flapping of the wall-detached jet, producing flow instabilities inside the steam turbine control valve.

The spatial patterns and frequency spectra of the mode coefficients for pressure POD modes of the scattered wall-detached-flow are illustrated in Fig. 14. Four energetic pressure POD modes show symmetric features along the centerline of the steam turbine control valve, which are different from the antisymmetric features of dominant velocity POD modes shown in Fig. 12. For mode 1, several discrete regions with intensified negative pressure fluctuations were identified from the valve throat to the upstream region of the valve pipe $\left(5.1 \leq y / D_{v} \leq 6.6\right)$, which are related to the PrandtlMayer expansion process of the disperse wall-detached jet. ${ }^{41}$ In addition, the massive positive pressure fluctuations at the bottom of the circular pipe $\left(1.2 \leq y / D_{v} \leq 3.3\right)$, along with discrete negative velocity fluctuations, constituted an axial pressure fluctuation mode. Mode 2 was also characterized by an axial spatial pattern. However, the regions with intensive pressure fluctuations moved upward to the top of the valve pipe. Also, the pressure fluctuations inside the valve spindle cavity were more intense than in mode 1 , which may have induced serious aerodynamic force fluctuations and even
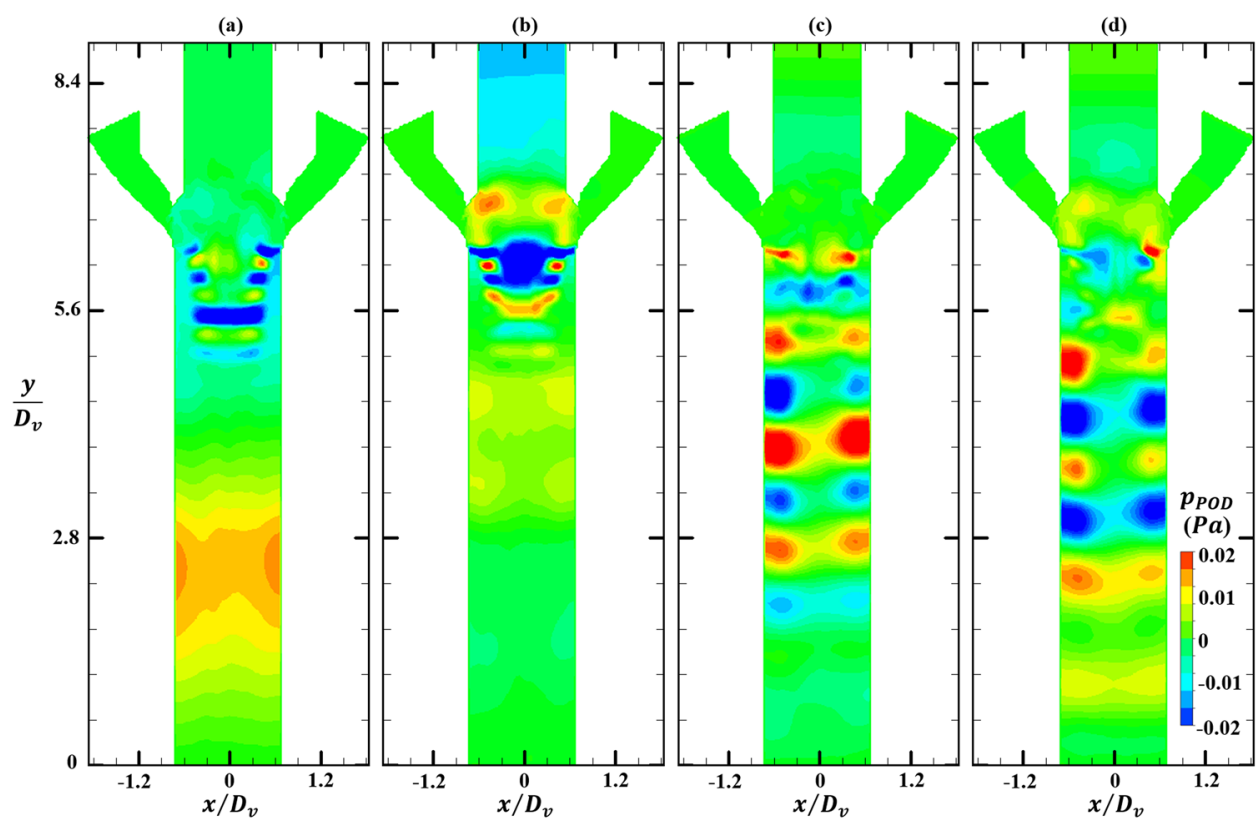

FIG. 14. Spatial patterns and the corresponding frequency spectra of the mode coefficients for pressure POD modes of the scattered wall-detached-flow $(\varepsilon=0.5, \pi=0.6)$ : (a) pressure POD mode 1 ; (b) pressure POD mode 2; (c) pressure POD mode 3 ; and (d) pressure POD mode 4.
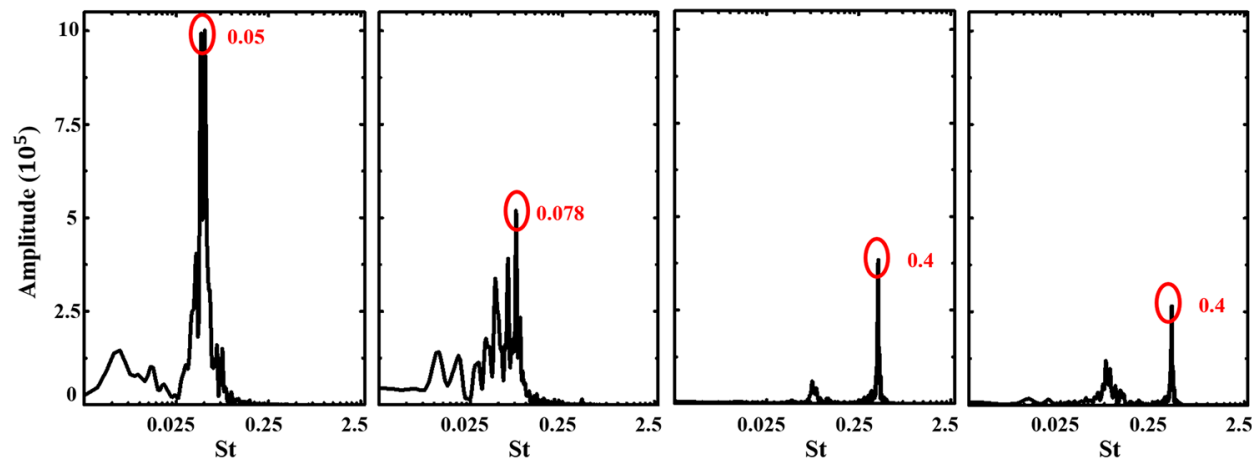
flow-induced vibrations to the valve spindle. A detailed inspection of modes 3 and 4 exhibited significantly different features from modes 1 and 2, especially the pressure fluctuations inside the valve pipe. A sequence of regions with alternating positive and negative pressure fluctuations were attached to the wall surfaces of the valve pipe, which would be attributed to the interactions between the boundary layer vortices and the surrounding reverse flow. ${ }^{37}$ In addition, the spatial patterns of modes 3 and 4 displayed an inverse cross correlation property, e.g., positive pressure fluctuations were identified in mode 3 at $3.4 \leq y / D_{v} \leq 4$, whereas negative pressure fluctuations were identified in mode 4 . These pressure fluctuations are related to the flow separations and reattachments buried in the interactions between the surrounding reverse flow and the disperse walldetached jet. Beyond that, lower frequencies at $S t=0.05$ and $S t=$ 0.078 were identified for modes 1 and 2, respectively, whereas a high frequency at $S t=0.4$ was identified for modes 3 and 4 . The low-frequency pressure fluctuations in modes 1 and 2 are primarily related to the unsteady flow behaviors, and the discrepancies from velocity modes are reasonable as the velocity field is a vector field, whereas the pressure field is a scalar field. However, the high-pressure fluctuations in modes 3 and 4 may also be the result of natural acoustic modes inside the valve pipe, ${ }^{42}$ yielding acoustic emissions and even acoustic fatigue to the valve pipe.

\section{Merged wall-detached-flow}

For the merged wall-detached-flow inside the steam turbine control valve, dominant velocity POD modes and the corresponding frequency spectra of their mode coefficients are illustrated in Fig. 15. A qualitative view of modes 1 and 2 demonstrates an antisymmetric spatial feature at regions with intensified positive and negative velocity fluctuations alternated inside the valve pipe. Simultaneously, large-scale vortex structures were identified between these alternating positive and negative velocity fluctuations. However, compared with dominant velocity modes of the scattered wall-detached-flow, there were evident differences in the spatial scales and locations of the intensive velocity fluctuations and the accompanying vortex structures. Due to the more intensified detachment scale of the central wall-detached jet of the merged wall-detached-flow, the horizontal spatial scales of the regions with intensive velocity fluctuations increased, whereas the vertical
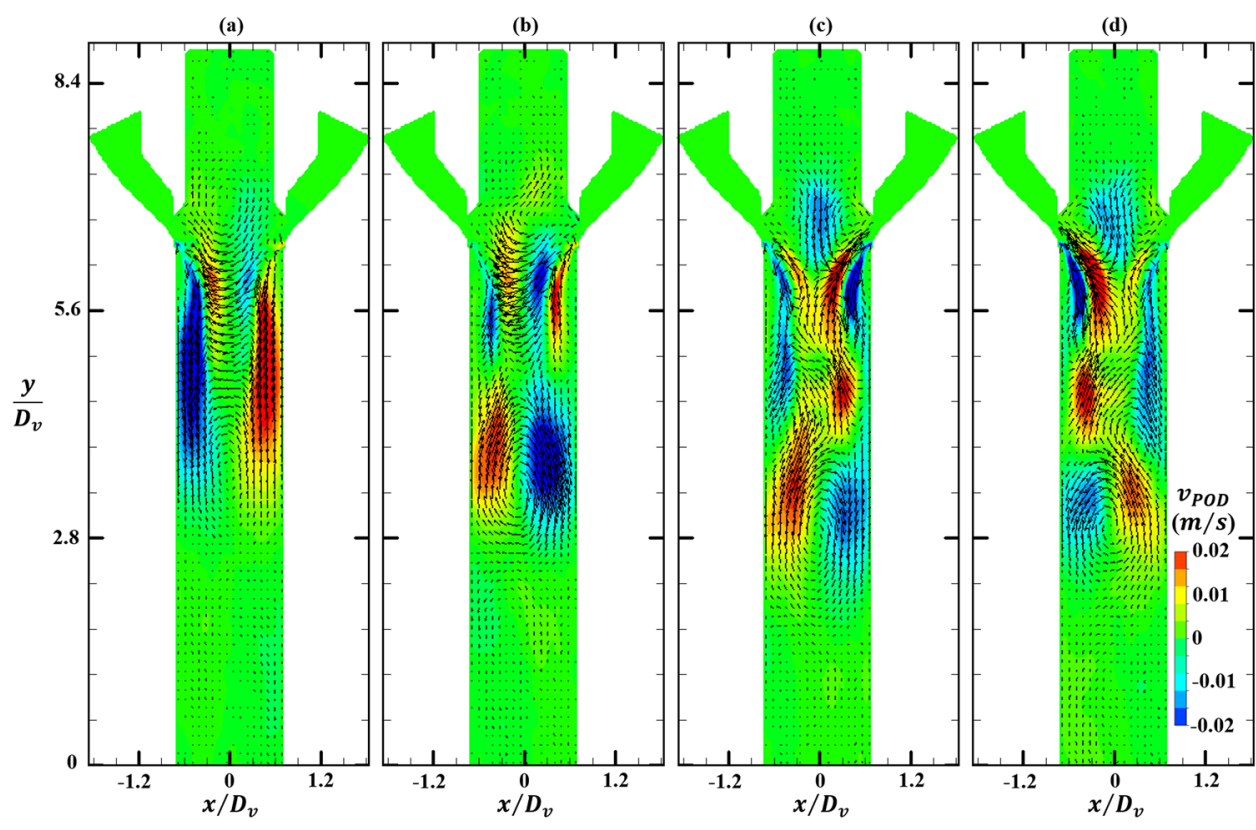

FIG. 15. Spatial patterns and the corresponding frequency spectra of the mode coefficients for velocity POD modes of the merged wall-detached-flow inside the steam turbine control valve $(\varepsilon=0.25$, $\pi=0.4$ ): (a) velocity POD mode $1 ;$ (b) velocity POD mode 2 ; (c) velocity POD mode 3; and (d) velocity POD mode 4.
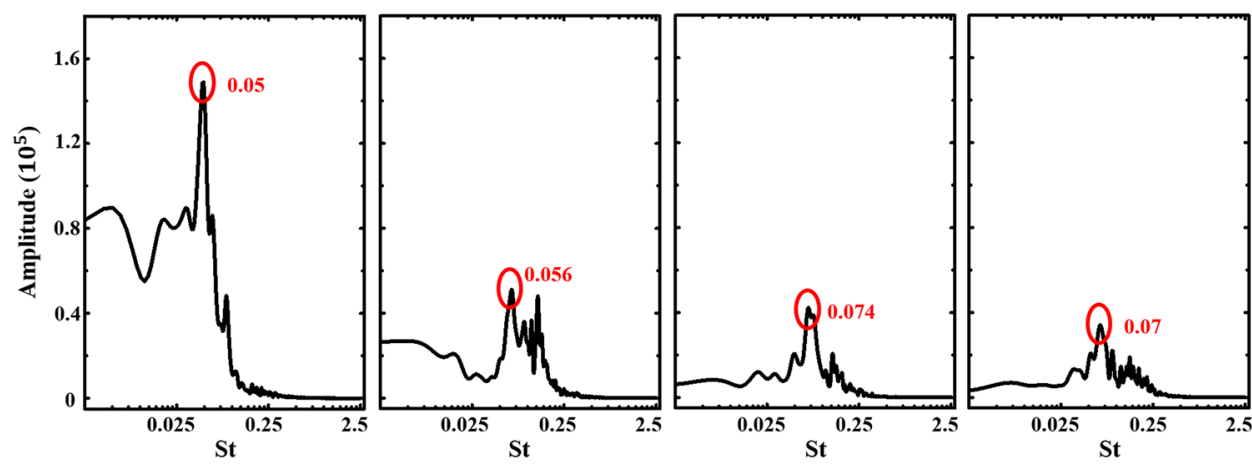
spatial scales decreased; therefore, these regions with intensive velocity fluctuations and the accompanying vortex structures moved upward to the top and middle of the valve pipe $\left(2.8 \leq y / D_{v} \leq 6.7\right)$. Moreover, even the vertical spatial scales of regions with intensive velocity fluctuations for mode 1 were larger than those of mode 2; the peak frequency of mode $1(S t=0.05)$ was quite close to that of mode $2(S t=0.056)$. Subsequently, modes 3 and 4 exhibited asymmetric spatial features and a peak frequency at $S t=0.07$. For mode 3 , the velocity fluctuations arising from the right side of the valve throat were more intense than those from the left side of the valve throat, yielding more unstable jet expansions at the right side of the valve pipe. For mode 4, this asymmetric phenomenon was reversed. The velocity fluctuations arising from the left side of the valve throat were more intense than those from the right side of the valve throat, yielding more unstable jet expansions at the left side of the valve pipe.

Subsequently, the first two velocity POD modes were used to reconstruct the flow field with the corresponding time-averaged flow field. Four successive phase-averaged flow fields with intervals of $t_{m} / 4$ and average bins of $\pm 5^{\circ}$ were determined and are illustrated in Fig. 16. Here, $t_{m}$ corresponds to the peak frequency at $S t=0.05$. A comparative view of Fig. 16 demonstrates extremely unstable flow behavior inside the steam turbine control valve, i.e., the horizontal flapping motion of the central wall-detached jet, the vertical out-phase oscillations of the surrounding reverse flow, and the cavity vortex flow. At $t=0$, the right jet exhibited more intensive expansion, and thus, the trailing edges of the jet expansion flapped toward the left side of the valve pipe. Simultaneously, the surrounding reverse flow $\left(3.9 \leq y / D_{v} \leq 6.5\right)$ at the right wall surfaces of the pipe and the vortex structure at the left entrance of the spindle cavity became considerably enlarged. At $t=\frac{t_{m}}{4}$, the left jet expansion intensified, whereas the right jet expansion became gradually attenuated, yielding a relatively symmetric flow pattern. Meanwhile, the right reverse flow and the right vortex structure at the cavity entrance attenuated, whereas the left reverse flow and the left vortex structure at the cavity entrance became obviously enlarged. Such intensified flow behavior at the left side and the attenuated flow behavior at the right side continued until $t=\frac{t_{m}}{2}$, when the global flow pattern became asymmetric again with the trailing edges of the jet expansion flapping to the right side of the valve pipe and the left reverse flow extending from $y / D_{v}=5.1$ to $y / D_{v}=3.8$. Finally, at $t=\frac{3 t_{m}}{4}$, the right jet exhibited more intensive expansion again, such as at $t=0$; however, the trailing edges of the jet expansion still flapped toward the right side of the circular pipe. Simultaneously, the left reverse flow became attenuated and returned to $y / D_{v}=5.1$, whereas the right reverse flow was enlarged from $y / D_{v}=5.5$ to $y / D_{v}=4.9$.

The energetic pressure POD modes and corresponding frequency spectra of the merged wall-detached-flow are illustrated Fig. 17. A global view of Fig. 17 demonstrates axial distribution with alternating positive and negative pressure fluctuations along the valve pipe. The regions with intensive pressure fluctuations were located primarily at the top and middle of the circular pipe $(2.8 \leq$ $\left.y / D_{v} \leq 6.7\right)$, results that coincided with those of the dominant velocity POD modes. For mode 1, a pair of concentrated regions with negative and positive pressure fluctuations were identified below the spindle cavity $\left(5.2 \leq y / D_{v} \leq 6.3\right)$, which are related to the merging of the central wall-detached jet. Subsequently, discrete regions with positive pressure fluctuations $\left(3.5 \leq y / D_{v} \leq 4.8\right)$ are attributed to the unstable expansions after the merging of the central walldetached jet. For mode 2, the evident differences from mode 1 were the essential negative pressure fluctuations located in the middle of

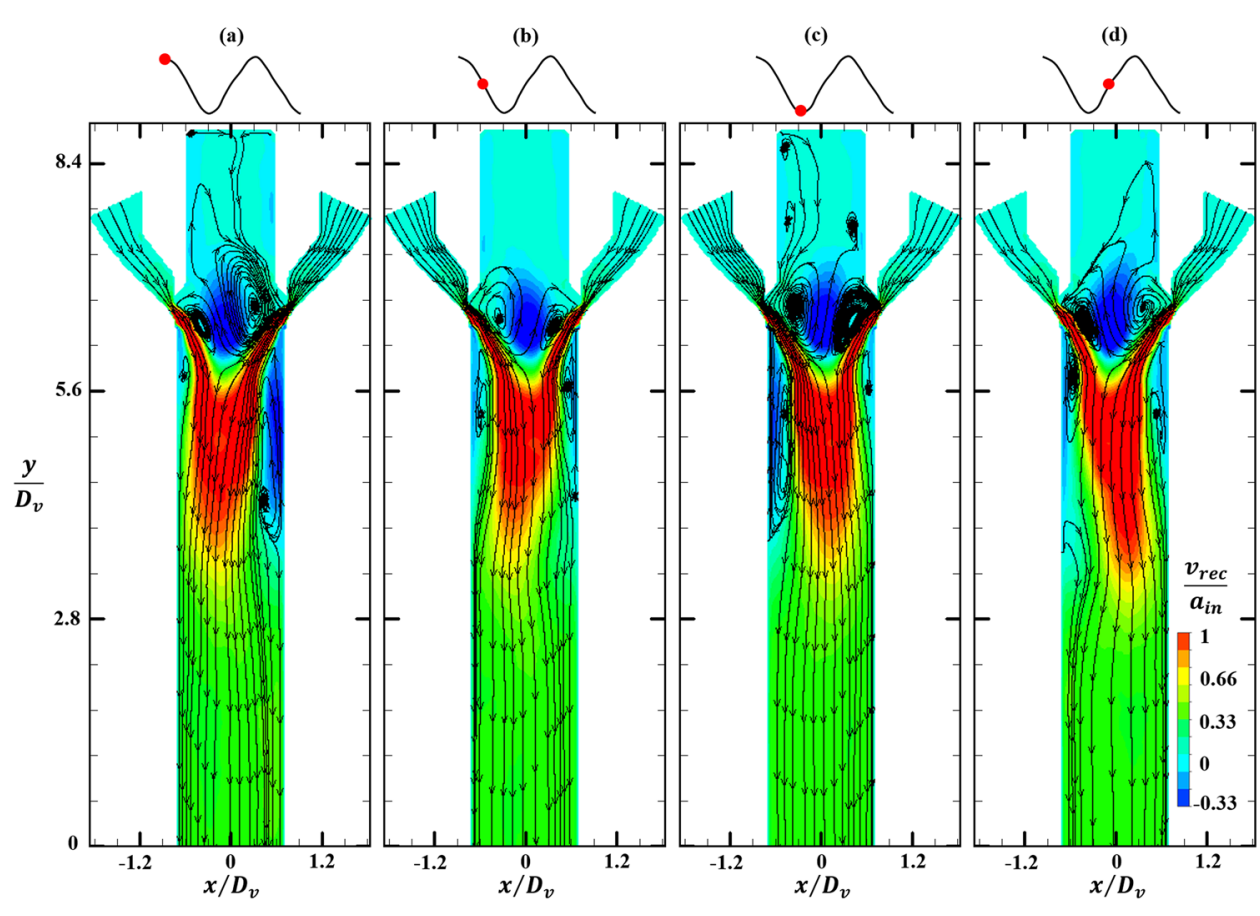

FIG. 16. Temporal evolutions of the reconstructed flow fields with the first two velocity POD modes of the merged walldetached-flow $(\varepsilon=0.25, \pi=0.4)$ : (a) $t=0$; (b) $t=t_{m} / 4$; (c) $t=t_{m} / 2$; and (d) $t=3 t_{m} / 4$. 

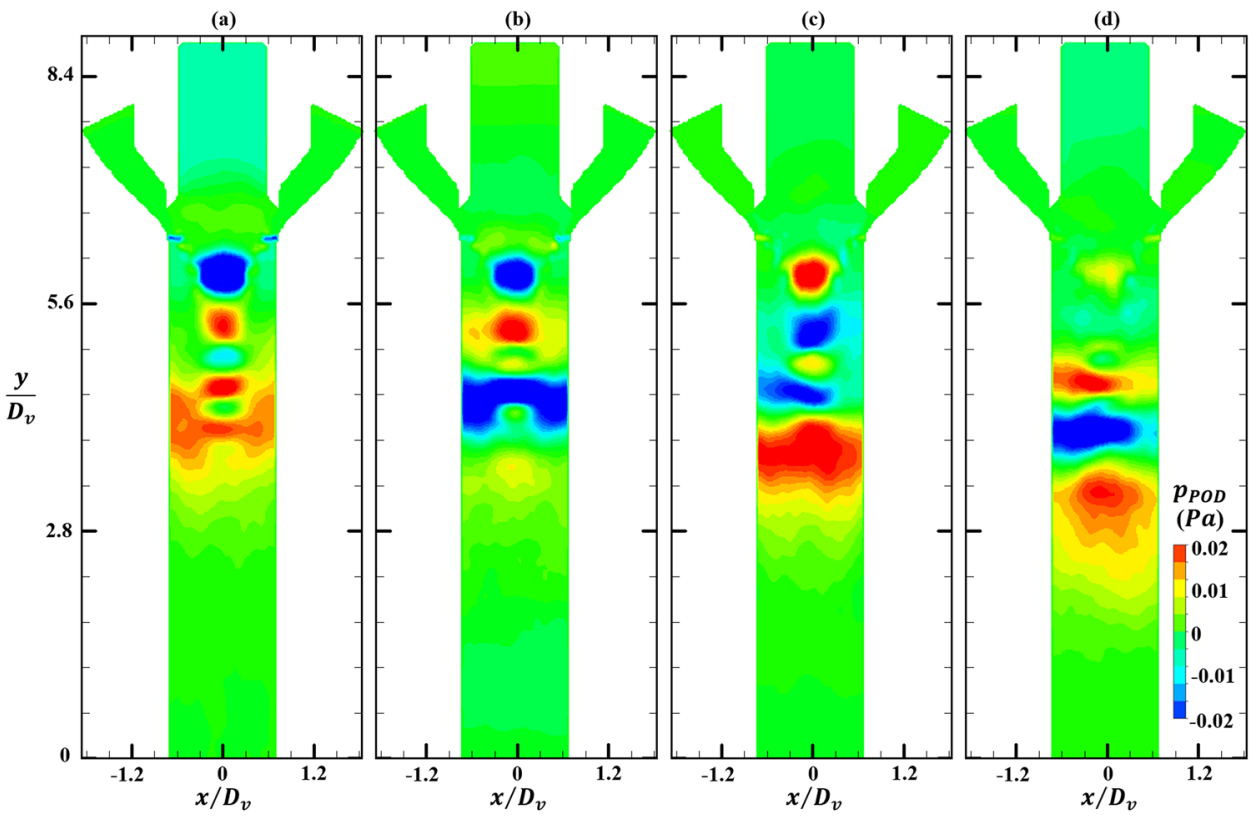

FIG. 17. Spatial patterns and the corresponding frequency spectra of the mode coefficients for the pressure POD modes of the merged wall-detached-flow $(\varepsilon=$ $0.25, \pi=0.4)$ : (a) pressure POD mode 1; (b) pressure POD mode 2; (c) pressure POD mode 3 ; and (d) pressure POD mode 4.
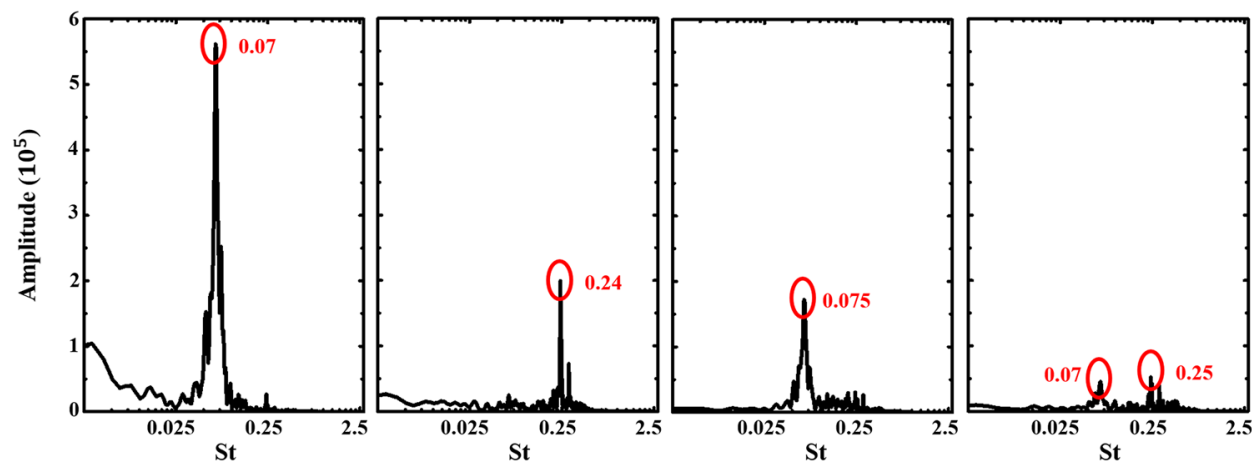

the circular pipe $\left(3.9 \leq y / D_{v} \leq 4.5\right)$. For mode 3, two pairs of concentrated regions with positive and negative pressure fluctuations were sequentially identified below the spindle cavity. For mode 4, the pressure fluctuations at the top of the circular pipe were quite attenuated, whereas the regions with intensive pressure fluctuations moved down to $2.4 \leq y / D_{v} \leq 5.1$, which is related to the unstable expansions after the mixing of the annular wall-detached jet. Moreover, a peak frequency around $S t=0.07$ was identified for modes 1 , 3 , and 4 . This low frequency closely coincided with that of velocity POD modes 3 and 4 . However, the high frequency at $S t=0.24$ for pressure POD mode 2 may combine with the axial acoustic mode inside the valve pipe ${ }^{37}$ and transfer to the upstream valve cavity and the downstream steam turbine chamber.

\section{Intersected wall-detached-flow}

The dominant velocity POD modes and the corresponding frequency spectra of their mode coefficients corresponding to intersected wall-detached-low are illustrated in Fig. 18. A comparative view of Fig. 18 demonstrates significantly different spatial patterns from the scattered wall-detached-flow and merged wall-detachedflow. For the intersected wall-detached-flow, the regions with intensive velocity fluctuations of these four modes were located mostly around the entrance of the spindle cavity. These intensive velocity fluctuations would interact with the reversed cavity vortex flow, act on the valve spindle, and yield fluctuated aerodynamic forces. However, the velocity fluctuations inside the valve pipe were extremely attenuated, indicating that the flow fields here were relatively stable. In addition, the intensive velocity fluctuations inside the valve pipe were located primarily along the pipe's centerline, represented by an antisymmetric feature. These velocity fluctuations would interact with the complex wave reflections, yielding unstable wave propagations inside the valve pipe. A comparative view of the frequency spectra of these four dominant modes' coefficients shows a peak frequency at $S t=0.086$ for the first two modes, whereas for modes 3 and 4, no obvious peak frequency was identified.

The first two velocity POD modes were used to reconstruct the flow fields to exhibit the unsteady behaviors of the intersected walldetached-flow. Intervals of $t_{i} / 4$ and average bins of $\pm 5^{\circ}$ were used to determine the successive phase-averaged flow fields, which are illustrated in Fig. 19. Here, $t_{i}$ corresponds to the peak frequency at $S t=0.086$. Global observation of Fig. 19 demonstrates common 

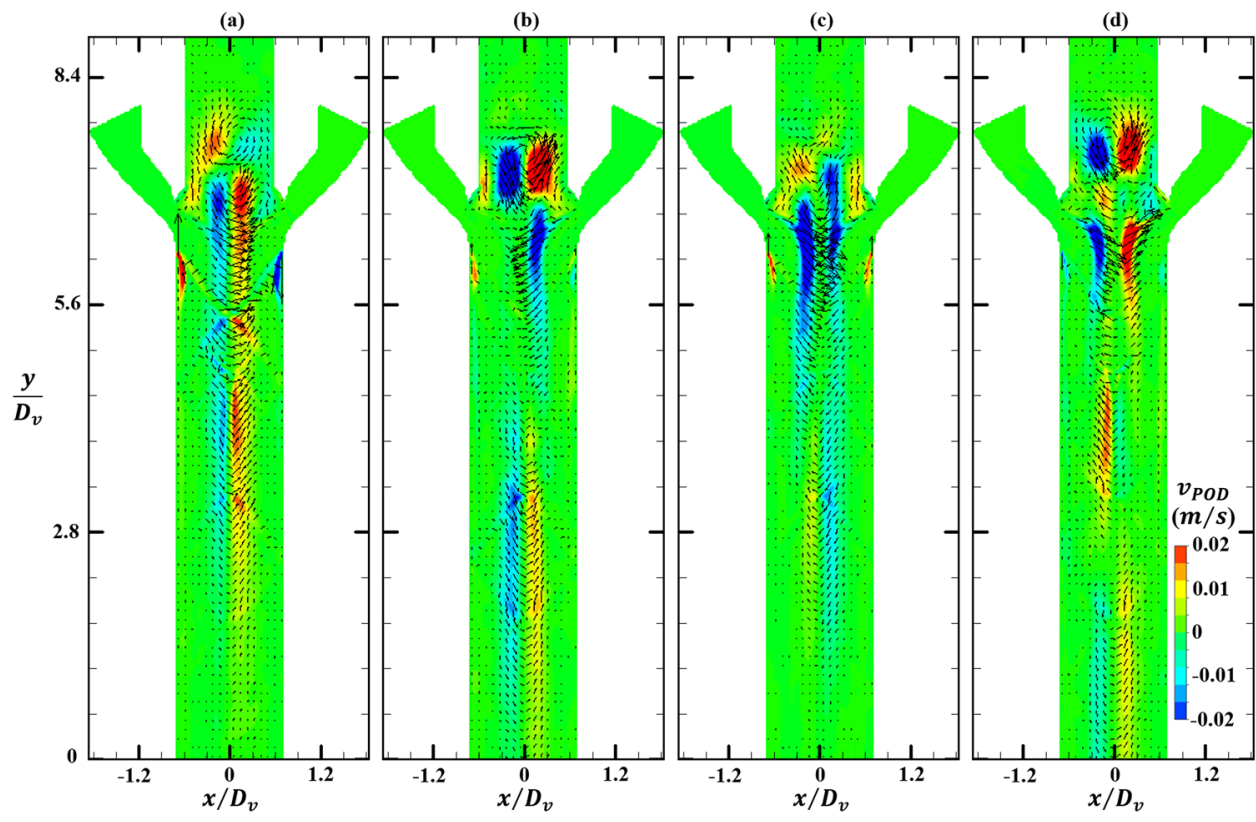

FIG. 18. Spatial patterns and the corresponding frequency spectra of the mode coefficients for the velocity POD modes of the intersected wall-detachedflow inside the steam turbine control valve $(\varepsilon=0.5, \pi=0.4)$ : (a) velocity POD mode 1 ; (b) velocity POD mode 2 ; (c) velocity POD mode 3 ; and (d) velocity POD mode 4.
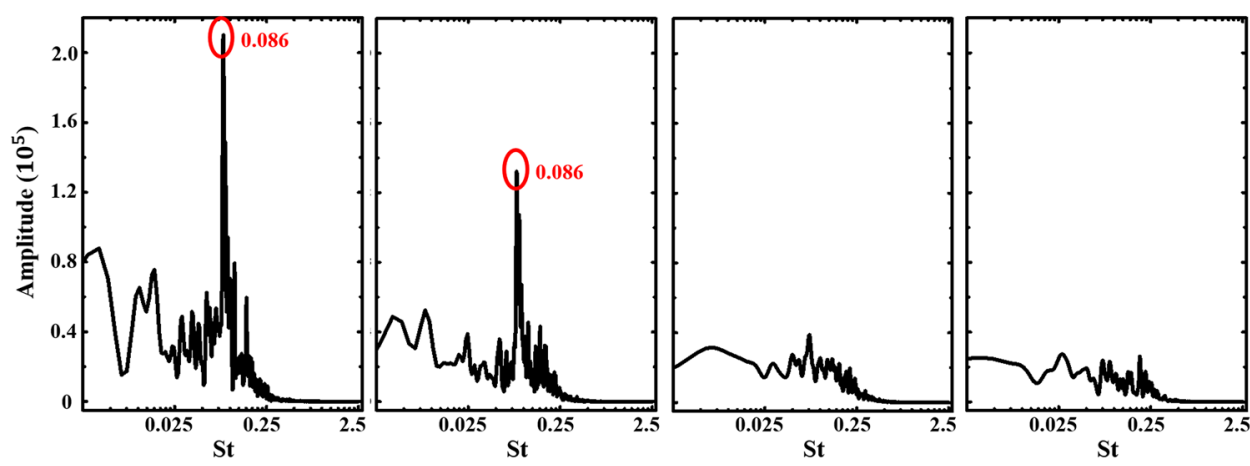

features of the reconstructed flow fields during those four moments, i.e., the unsteady oscillations of the reversed cavity vortex flow and the steady motion of the flow field inside the circular pipe. For the unsteady cavity vortex flow, the intensification and attenuation of the vortex structures were identified from a comparative view of Fig. 19. The spatial scale of the left vortex structure gradually increased from $t=0$ to $t=\frac{t_{i}}{2}$, becoming attenuated at $t=\frac{3 t_{i}}{4}$. Meanwhile and oppositely, the spatial scale of the right vortex structure gradually decreased from $t=0$ to $t=\frac{t_{i}}{2}$ and then intensified at $t=\frac{3 t_{i}}{4}$. Such alternating variations would induce the transverse instability of the valve spindle. For the flow field inside the circular pipe, even though the temporal evolution was quite stable, the spatial velocity variations were extremely discontinuous due to the complex wave reflections along the wall surfaces of the circular pipe. Such phenomenon with velocity discontinuities induced by wave reflections can also be identified in the study by Soni and De. ${ }^{3}$ For example, an obvious velocity discontinuity was identified near the location of $y / D_{v}=4.8$; upstream of this location, the vertical velocity almost reached $v_{r e c} / a_{i n}=1.67$; however, downstream of this location, the vertical velocity collapsed to only $v_{r e c} / a_{i n}=0.81$. Subsequently, the vertical velocity gradually recovered along with the wave reflections inside the valve pipe. Such a velocity drop and recovery are closely related to the expansion and recompression of the central wall-detached jet, constituting a complex Prandtl-Mayer expansion process inside the valve pipe.

Finally, energetic pressure POD modes and the corresponding frequency spectra of their mode coefficients for the intersected wall-detached-flow are displayed in Fig. 20. A global view of Fig. 20 shows spatial patterns similar to the RMS of pressure fluctuations of such intersected wall-detached flow [Fig. 10(d)]. A series of diagonal crosslines with alternating positive and negative pressure fluctuations were identified along the circular pipe, which represented the complex wave reflections on the wall surfaces and wave propagation inside the valve pipe. ${ }^{34,35}$ Moreover, a peak frequency at $S t=0.089$ was identified for the first two pressure POD modes, which is almost equal to that of the first two velocity POD modes. Such a phenomenon confirms the strong modulation by the shockwave reflections, which would essentially dominate the velocity field and pressure field with the same fluctuation frequency. However, a comparative view of the first two pressure modes demonstrated an antisymmetric feature of the pressure fluctuation distribution. For example, at $4.5 \leq y / D_{v} \leq 6.2$ of mode 1 , negative pressure fluctuations 


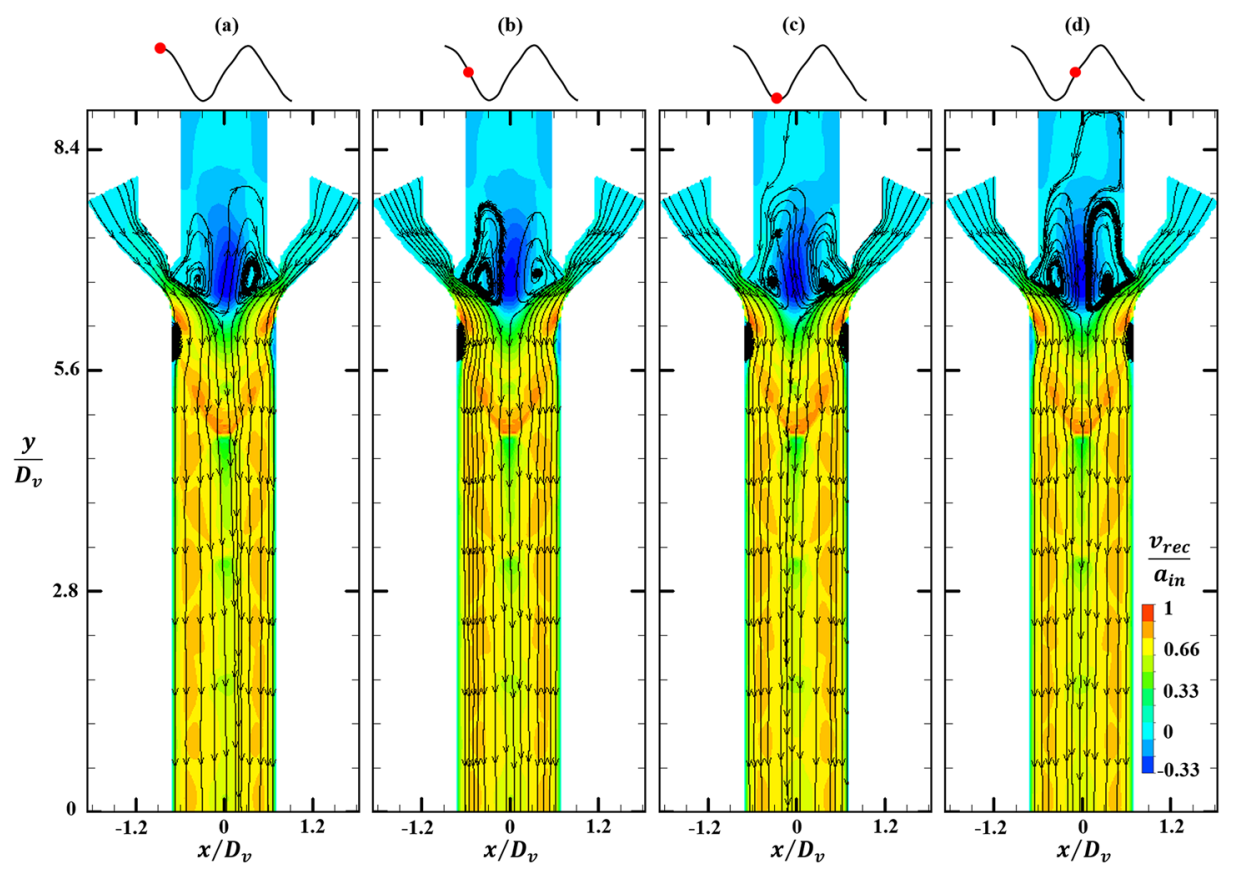

FIG. 19. Temporal evolutions of the reconstructed flow field with the first two velocity POD modes of the intersected wall-detached-flow $(\varepsilon=0.5, \pi=0.4)$ : (a) $t=0$; (b) $t=t_{i} / 4$; (c) $t=t_{i} / 2$; and (d) $t=$ $3 t_{i} / 4$.

were identified from the diagonal crossline from the left pipe edge to the right pipe edge along the streamwise direction; oppositely for the diagonal crossline from the right pipe edge to the left pipe edge, the pressure fluctuations are positive. Such alternating variations may induce asymmetric shock-wave reflections inside the circular pipe. However, a detailed inspection of modes 3 and 4 exhibited a quasisymmetric feature of the pressure fluctuation distributed inside the middle section of the circular pipe.

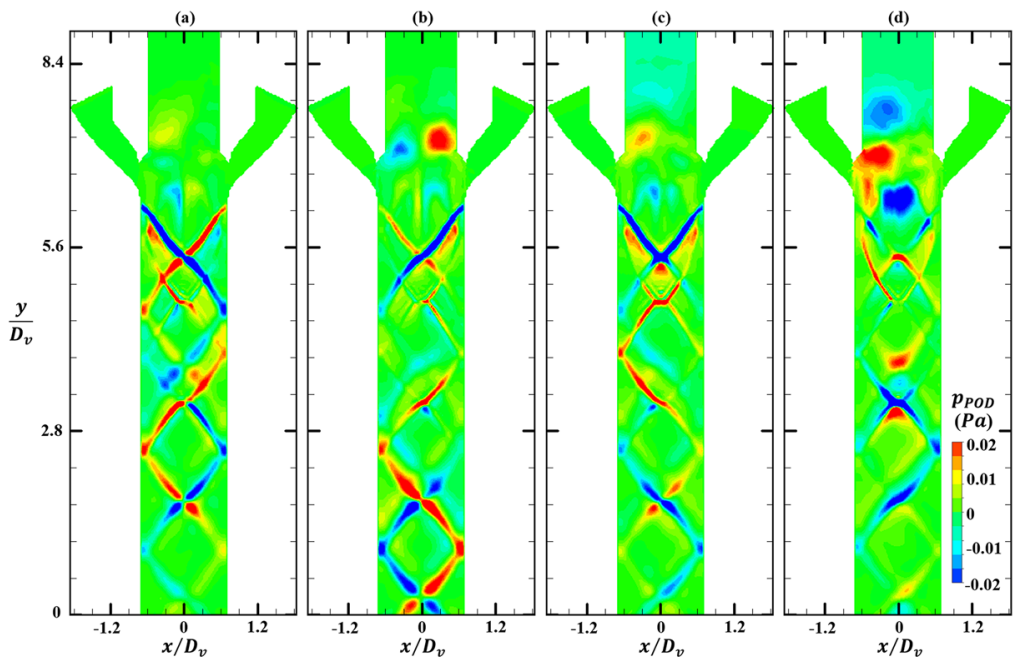

FIG. 20. Spatial patterns and the corresponding frequency spectra of the mode coefficients for pressure POD modes of the intersected wall-detached-flow $(\varepsilon=0.5, \pi=0.4)$ : (a) pressure POD mode 1; (b) pressure POD mode 2; (c) pressure POD mode 3; and (d) pressure POD mode 4.

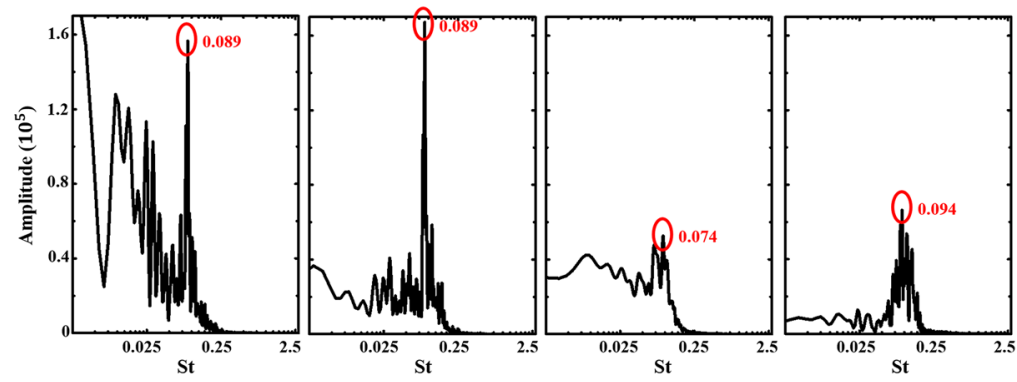




\section{CONCLUSIONS}

The wall-detached flow inside a control valve located before an ultra-supercritical steam turbine was comprehensively investigated with DES, POD, and the flow reconstruction method. The dependency of the wall-detached flow on the control valve's opening ratio $(0.2 \leq \varepsilon \leq 0.6)$ and pressure ratio $(0.2 \leq \pi \leq 0.85)$ was first established by $2 \mathrm{D}$ steady-state SST simulations. By conducting $3 \mathrm{D}$ transient-state DESs, three types of wall-detached flow, i.e., scattered wall-detached-flow ( $\varepsilon=0.5, \pi=0.6$ ), merged wall-detached-flow $(\varepsilon=0.25, \pi=0.4)$, and intersected wall-detached-flow ( $\varepsilon=0.5$, $\pi=0.4$ ), were then identified by distinguishing the detachment scale of the central wall-detached jet. Meanwhile, DES on the wallattached flow ( $\varepsilon=0.25, \pi=0.6$ ) was also conducted for comparison. The major findings of this study are as follows:

(i) Comparisons of the statistical flow quantities among the different wall-detached flow patterns demonstrated that the scattered wall-detached-flow presented a relatively stable flow feature, whereas the merged wall-detached-flow exhibited the most intensive velocity and pressure fluctuations inside the circular pipe of the steam turbine control valve; the intersected wall-detached-flow would encounter serious shock-wave reflections along the downstream pipe.

(ii) For the scattered wall-detached-flow, the POD analysis and flow reconstruction on the velocity field exhibited considerable velocity fluctuations attached to the wall surfaces of the valve pipe, interacting with the scattered walldetached jet and inducing horizontal flapping motion at the trailing edges of the jet expansion. In addition, pressure POD modes exhibited an axial symmetric feature. Multiple small-scale regions with alternating positive and negative pressure fluctuations coincided with the flow separations and reattachments buried in the interactions between the surrounding reverse flow and the central wall-detached jet.

(iii) For the merged wall-detached-flow, by conducting POD analysis and flow reconstruction on the velocity field, the dominant flow structures were determined. These dominant flow structures were represented by vertical out-phase oscillation and the horizontal flapping motion of the central walldetached jet, along with out-phase oscillations of the surrounding reverse flow and reversed cavity vortex flow. By conducting POD analysis on the pressure field, large-scale regions with axial-alternating positive and negative pressure fluctuations were closely related to the unstable expansions of the converged jet flow.

(iv) For the intersected wall-detached-flow, the POD analysis found that the complex shock-wave reflections along the wall surfaces of the valve pipe would modulate the dominant vortex structures and energetic pressure fluctuation modes with a similar fluctuated frequency $(\mathrm{St}=0.086)$. Massive discontinuities of the reconstructed velocity field and a series of diagonal crosslines with intensive pressure fluctuations were identified, along with the shock-wave reflections and propagations inside the valve pipe.

These findings are of great practical significance for the operation and optimization of steam turbine control valves in thermal power plants. Particularly, operations with merged wall-detachedflow, which generally appear under larger opening ratios and intermediate pressure ratios, should be prevented. In addition, the natural frequency of the valve system should be designed far away from the frequency with vertical out-phase oscillations and horizontal flapping motion of the central wall-detached jet.

\section{ACKNOWLEDGMENTS}

The authors gratefully acknowledge the financial support received from the Ministry of Science and Technology of the People's Republic of China (Grant No. 2017YFB0602103) for this study.

\section{REFERENCES}

${ }^{1}$ P. Kriesels, M. Peters, A. Hirschberg, A. Wijnands, A. Iafrati, G. Riccardi, and J. Bruggeman, "High amplitude vortex-induced pulsations in a gas transport system," J. Sound Vib. 184(2), 343-368 (1995).

${ }^{2}$ S. Dequand, X. Luo, J. Willems, and A. Hirschberg, "Helmholtz-like resonator self-sustained oscillations, part 1: Acoustical measurements and analytical models," AIAA J. 41(3), 408-415 (2003).

${ }^{3}$ J. Hardin, F. Kushner, and S. Koester, "Elimination of flow induced instability from steam turbine control valve," in Proceedings of the 32nd Turbomachinery Symposium (Texas A\&M University, Turbomachinery Laboratories, 2003), pp. 99-109.

${ }^{4}$ S. Michaud, S. Ziada, and H. Pastorel, "Acoustic fatigue of a steam dump pipe system excited by valve noise,” J. Pressure Vessel Technol. 123(4), 461-468 (2001).

${ }^{5}$ M. Nakano, E. Outa, and K. Tajima, "Aerodynamic study on noise and vibration generated in high pressure gas valves: Part 1: Flow patterns and noise of supersonic air flow discharged through conical valve plugs into atmosphere," Bull. JSME 22(173), 1578-1586 (1979).

${ }^{6} \mathrm{M}$. Nakano and K. Tajima, "Aerodynamic study on noise and vibration generated in high pressure gas valves: Part 1: Noise and vibration induced by internal oscillating flows in conical plug-circular chest valves," Bull. JSME 27(226), 691-699 (1984).

${ }^{7}$ M. Pluviose, “Stabilization of flow through steam-turbine control valves," J. Eng. Gas Turbines Power 111(4), 642 (1989).

${ }^{8}$ D. Zhang, A. Engeda, J. R. Hardin, and R. H. Aungier, "Experimental study of steam turbine control valves," Proc. - Inst. Mech. Eng. 218(5), 493-508 (2004).

${ }^{9}$ D. Zhang and A. Engeda, "Venturi valves for steam turbines and improved design considerations," Proc. - Inst. Mech. Eng., Part A 217(2), 219-230 (2003).

${ }^{10}$ L. Zeng, G. Liu, J. Mao, S. Wang, Q. Yuan, H. Yuan, and Y. Xu, "Flow-induced vibration and noise in control valve," Proc. - Inst. Mech. Eng., Part C 229(18), 3368-3377 (2015).

${ }^{11}$ C. Domnick, D. Brillert, C. Musch, and F. K. Benra, "Clarifying the physics of flow separations in steam turbine inlet valves at part load operation and improved design considerations," J. Fluids Eng. 139, 081105-1 (2017).

${ }^{12}$ P. Wang, H. Ma, B. Quay, D. A. Santavicca, and Y. Liu, "Computational fluid dynamics of steam flow in a turbine control valve with a bell-shaped spindle," Appl. Therm. Eng. 129, 1333-1347 (2018).

${ }^{13}$ R. Morita, F. Inada, M. Mori, K. Tezuka, and Y. Tsujimoto, "CFD simulations and experiments of flow fluctuations around a steam control valve," J. Fluids Eng. 129(1), 48-54 (2007).

${ }^{14}$ G. Zanazzi, O. Schaefer, and M. Sell, "Unsteady CFD simulation of control valve in throttling conditions and comparison with experiments," in ASME Turbo Expo 2013: Turbine Technical Conference and Exposition (ASME, 2013), p. V05BT25A019.

${ }^{15} \mathrm{~K}$. Yonezawa, R. Ogawa, and K. Ogi, "Flow-induced vibration of a steam control valve," J. Fluid Struct. 35, 76-88 (2012).

${ }^{16}$ L. Zeng, G. Liu, J. Mao, S. Wang, C. Zhang, and X. Yu, "Research on the coupling mechanism between alternating flow pattern and valve stem system of steam turbine control valve," in Volume 1B: Marine; Microturbines, Turbochargers and Small Turbomachines; Steam Turbines, 2014. 
${ }^{17}$ L. Zeng and G. Liu, "A novel numerical simulation method to verify turbulence models for predicting flow patterns in control valves," J. Fluid Sci. Technol. 10(1), JFST0007 (2015).

${ }^{18}$ C. Domnick, F. Benra, and D. Brillert, "Numerical investigation on the timevariant flow field and dynamic forces acting in steam turbine inlet valves," J. Eng. Gas Turbines Power 137(8), 081601 (2015).

${ }^{19}$ C. Domnick, F. Benra, D. Brillert, H. Dohmen, and C. Musch, "Investigation on flow-induced vibrations of a steam turbine inlet valve considering fluid-structure interaction effects," J. Eng. Gas Turbines Power 139(2), 022507 (2016).

${ }^{20} \mathrm{P}$. Wang and Y. Liu, "Unsteady flow behavior of a steam turbine control valve in the choked condition: Field measurement, detached eddy simulation and acoustic modal analysis," Appl. Therm. Eng. 117, 725-739 (2017).

${ }^{21} \mathrm{P}$. Wang, H. Ma, and Y. Liu, "Unsteady behaviors of steam flow in a control valve with T-junction discharge under the choked condition: Detached eddy simulation and proper orthogonal decomposition," J. Fluids Eng. 140(8), 081104-1-081104-13 (2018).

${ }^{22} \mathrm{P}$. Wang, H. Ma, and Y. Liu, "Proper orthogonal decomposition and extendedproper orthogonal decomposition analysis of pressure fluctuations and vortex structures inside a steam turbine control valve," J. Eng. Gas Turbines Power 141(4), 041035 (2019).

${ }^{23}$ R. Akkermans, A. Cieslik, L. Kamp, R. Trieling, H. Clercx, and G. Heijst, "The three-dimensional structure of an electromagnetically generated dipolar vortex in a shallow fluid layer," Phys. Fluids 20(11), 116601 (2008).

${ }^{24}$ R. Viswanathan, J. Henry, J. Tanzosh, G. Stanko, J. Shingledecker, B. Vitalis, and R. Purgert, "US program on materials technology for ultra-supercritical coal power plants," J. Mater. Eng. Perform. 14(3), 281-292 (2005).

${ }^{25} \mathrm{P}$. Spalart, W. Jou, M. Strelets, and S. Allmaras, "Comments on the feasibility of LES for wings and on a hybrid RANS/LES approach," in 1st AFOSR International Conference on DNS/LES, 1997.

${ }^{26} \mathrm{M}$. Strelets, "Detached eddy simulation of massively separated flows," in 39th Aerospace Sciences Meeting and Exhibit, 2001.

${ }^{27}$ W. Wagner and H. Kretzschmar, "IAPWS industrial formulation 1997 for the thermodynamic properties of water and steam," in International Steam Tables: Properties of Water and Steam Based on the Industrial Formulation IAPWS-IF97 (Springer, 2008), pp. 7-150, see https://link.springer.com/chapter/10.1007/978-3 -540-74234-0_3.

${ }^{28}$ ANSYS, Inc., ANSYS CFX-Solver Theory Guide, Release 16.0, 2015.
${ }^{29} \mathrm{~B}$. Newman, "The deflection of plane jets by adjacent boundary layers-Coandă effect," in Boundary Layer and Flow Control, edited by G. V. Lachmann (Pergamon Press, New York, 1961).

${ }^{30}$ A. Kizilos and R. Rose, Experimental Investigations of Flight Control Surfaces Using Modified Air Jets (Honeywell, Inc., 1968), Doc. 12055-FRI.

${ }^{31}$ C. Lubert, "On some recent applications of the Coandă effect to acoustics," J. Acoust. Soc. Am. 128(4), 2286 (2010).

${ }^{32}$ A. Pophali, M. Bussmann, and H. Tran, "Supersonic jet impingement on a cylinder and characterization of the resulting deflected jets," J. Fluids Eng. 136(11), 111103-1-111103-11 (2014).

${ }^{33}$ A. Kastengren and J. Dutton, "Aspects of shear layer unsteadiness in a threedimensional supersonic wake,” J. Fluids Eng. 127(6), 1085-1094 (2005).

${ }^{34} \mathrm{P}$. Winroth and P. Alfredsson, "On shock structures in dynamic exhaust valve flows," Phys. fluids 31(2), 026107 (2019).

${ }^{35}$ R. Kumar and G. Rajesh, "Physics of vacuum generation in zero-secondary flow ejectors," Phys. Fluids 30(6), 066102 (2008).

${ }^{36}$ V. Vassiliev, S. Irmisch, M. Claridge, and D. Richardson, "Experimental and numerical investigation of the impact of swirl on the performance of industrial gas turbines exhaust diffusers," in Volume 6: Turbo Expo 2003, Parts A and B, 2003.

${ }^{37} \mathrm{R}$. Soni and A. De, "Role of jet spacing and strut geometry on the formation of large scale structures and mixing characteristics," Phys. Fluids 30, 056103 (2018).

${ }^{38} \mathrm{~J}$. Lumley, Stochastic Tools in Turbulence (Dover Publications, NY, USA, 2007).

${ }^{39} \mathrm{~L}$. Sirovich, "Turbulence and the dynamics of coherent structures. I. Coherent structures," Q. Appl. Math. 45, 561-571 (1987); "Turbulence and the dynamics of coherent structures. II. Symmetries and transformations," 45, 573 (1987); "Turbulence and the dynamics of coherent structures. III. Dynamics and scaling," 45, 583 (1987).

${ }^{40}$ B. Oudheusden, F. Scarano, N. Hinsberg, and D. Watt, "Phase-resolved characterization of vortex shedding in the near wake of a square-section cylinder at incidence," Exp. Fluids 39(1), 86-98 (2005).

${ }^{41} \mathrm{~J}$. Appleton, "Structure of a Prandtl-Meyer expansion in an ideal dissociating gas," Phys. Fluids 6(8), 1057-1062 (1963).

${ }^{42}$ E. Naudascher and D. Rockwell, Flow Induced Vibrations: An Engineering Guide (Dover Publications, Mineola, NY, 1994). 